\title{
Melnikov theory for weakly coupled nonlinear RLC circuits
}

Flaviano Battelli ${ }^{1 *}$ and Michal Fečkan ${ }^{2,3}$

Dedicated to Professor Ivan Kiguradze

*Correspondence:

battelli@dipmat.univpm.it

'Department of Industrial

Engineering and Mathematical

Sciences, Marche Polytecnic

University, Via Brecce Bianche 1,

Ancona, 60131, Italy

Full list of author information is

available at the end of the article

\begin{abstract}
We apply dynamical system methods and Melnikov theory to study small amplitude perturbation of some coupled implicit differential equations. In particular we show the persistence of such orbits connecting singularities in finite time provided a Melnikov like condition holds. Application is given to coupled nonlinear RLC system. MSC: Primary 34A09; secondary 34C23; 37G99
\end{abstract}

Keywords: implicit ode; perturbation; Melnikov method; RLC circuits

\section{Introduction}

In [1], motivated by [2, 3], the equation modeling nonlinear RLC circuits

$$
(u+f(u))^{\prime \prime}+\varepsilon \gamma(u+f(u))^{\prime}+u+\varepsilon h(t+\alpha, u, \varepsilon)=0
$$

has been studied. It is assumed that $f(u)$ and $h(t, u, \varepsilon)$ are smooth functions with $f(u)$ at least quadratic at the origin and satisfying suitable assumptions. Setting $v=(u+f(u))^{\prime}$ the equation reads

$$
\begin{aligned}
& \left(1+f^{\prime}(u)\right) u^{\prime}=v, \\
& v^{\prime}=-u-\varepsilon[h(t+\alpha, u, \varepsilon)+\gamma v] .
\end{aligned}
$$

It is assumed that, for some $u_{0} \in \mathbb{R}$, we have $f^{\prime}\left(u_{0}\right)+1=0$ and $u_{0} f^{\prime \prime}\left(u_{0}\right)<0$. So for $\varepsilon=0$ (2) has the Hamiltonian

$$
\mathcal{H}(u, v)=v^{2}+2 \int_{u_{0}}^{u} \sigma\left(1+f^{\prime}(\sigma)\right) d \sigma
$$

passing through $\left(u_{0}, 0\right)$. Clearly $\nabla \mathcal{H}\left(u_{0}, 0\right)=\left(\begin{array}{l}0 \\ 0\end{array}\right)$ and the Hessian of $\mathcal{H}$ at $\left(u_{0}, 0\right)$ is

$$
H_{\mathcal{H}}\left(u_{0}, 0\right)=\left(\begin{array}{cc}
2 u_{0} f^{\prime \prime}\left(u_{0}\right) & 0 \\
0 & 2
\end{array}\right),
$$

O2014 Battelli and Fečkan; licensee Springer. This is an Open Access article distributed under the terms of the Creative Commons Attribution License (http://creativecommons.org/licenses/by/2.0), which permits unrestricted use, distribution, and reproduction in any medium, provided the original work is properly cited. 
so that the condition $u_{0} f^{\prime \prime}\left(u_{0}\right)<0$ means that $\left(u_{0}, 0\right)$ is a saddle for $\mathcal{H}$. Multiplying the second equation by $1+f^{\prime}(u)$ we get the system

$$
\left(1+f^{\prime}(u)\right)\left(\begin{array}{c}
u^{\prime} \\
v^{\prime}
\end{array}\right)=\left(\begin{array}{c}
v \\
-\left(1+f^{\prime}(u)\right)\{u+\varepsilon[h(t+\alpha, u, \varepsilon)+\gamma v]\}
\end{array}\right) .
$$

Note that (2) falls in the class of implicit differential equations (IODE) like

$$
A(x) x^{\prime}=f(x)+\varepsilon h(t, x, \varepsilon, \kappa), \quad(\varepsilon, \kappa) \in \mathbb{R} \times \mathbb{R}^{m},
$$

with $A(u, v)=\left(\begin{array}{cc}1+f^{\prime}(u) & 0 \\ 0 & 1\end{array}\right)$. Obviously, $\operatorname{det} A(u, v)=1+f^{\prime}(u)$ vanishes on the line $\left(u_{0}, v\right)$ and the condition $f^{\prime \prime}\left(u_{0}\right) \neq 0$ implies that the line $u=u_{0}$ consists of noncritical 0 -singularities for (3) (see [4, p.163]). Let $\mathcal{N} L$ denote the kernel of the linear map $L$ and $\mathcal{R} L$ its range. Then $\mathcal{R} A\left(u_{0}, 0\right)$ is the subspace having zero first component and then the right hand side of (3) belongs to $\mathcal{R} A\left(u_{0}, 0\right)$ if and only if $v=0$. So all the singularities $\left(u_{0}, v\right)$ with $v \neq 0$ are impasse points while $\left(u_{0}, 0\right)$ is a so called $I$-point (see [4, pp.163-166]). Quasilinear implicit differential equations, such as (4), find applications in a large number of physical sciences and have been studied by several authors [4-12]. On the other hand, there are many other works on implicit differential equations [13-18] dealing with more general implicit differential systems by using analytical and topological methods.

Passing from (2) to (3), in the general case, it corresponds to multiplying (4) by the adjugate matrix $A^{a}(x)$ :

$$
\omega(x) x^{\prime}=A^{a}(x)[f(x)+\operatorname{ch}(t, x, \varepsilon, \kappa)],
$$

where $\omega(x)=\operatorname{det} A(x)$. Here we note that $A$ and $x$ may have different dimensions in this paper depending on the nature of the equation but the concrete dimension is clear from that equation, so we do not use different notations for $A$ and $x$. Basic assumptions in [1] are $\omega\left(x_{0}\right)=0, \omega^{\prime}\left(x_{0}\right) \neq 0$ and $A^{a}\left(x_{0}\right) f\left(x_{0}\right)=0, A^{a}\left(x_{0}\right) h\left(t, x_{0}, \varepsilon, \kappa\right)=0$ for some $x_{0}$ (that is, $x_{0}$ is an $I$-point for (4)) and the existence of a solution $x(t)$ in a bounded interval $J$ tending to $x_{0}$ as $t$ tends to the endpoints of $J$.

It is well known $[4,8]$ that $\omega\left(x_{0}\right)=0$ and $\omega^{\prime}\left(x_{0}\right) \neq 0$ imply

$$
\operatorname{dim} \mathcal{N} A\left(x_{0}\right)=1, \quad \mathcal{R} A^{a}\left(x_{0}\right)=\mathcal{N} A\left(x_{0}\right), \quad \text { and } \quad \mathcal{N} A^{a}\left(x_{0}\right)=\mathcal{R} A\left(x_{0}\right),
$$

and then $A^{a}\left(x_{0}\right) f\left(x_{0}\right)=0$ is equivalent to the fact that $f\left(x_{0}\right) \in \mathcal{R} A\left(x_{0}\right)$.

Let $F(x):=A^{a}(x) f(x)$. It has been proved in [19] that (5) implies that rank $F^{\prime}\left(x_{0}\right)$ is at most 2. So, if $x \in \mathbb{R}^{n}$, with $n>2$ then $x=x_{0}$ cannot be hyperbolic for the map $x \mapsto F^{\prime}\left(x_{0}\right) x$.

In this paper we study coupled IODEs such as

$$
\begin{aligned}
& A_{0}\left(x_{1}\right) x_{1}^{\prime}=f\left(x_{1}\right)+\varepsilon g_{1}\left(t, x_{1}, x_{2}, \varepsilon, \kappa\right), \\
& A_{0}\left(x_{2}\right) x_{2}^{\prime}=f\left(x_{2}\right)+\varepsilon g_{2}\left(t, x_{1}, x_{2}, \varepsilon, \kappa\right),
\end{aligned}
$$

with $x_{1}, x_{2} \in \mathbb{R}^{2}$, $\operatorname{det} A_{0}\left(x_{0}\right)=0 \neq\left(\operatorname{det} A_{0}\right)^{\prime}\left(x_{0}\right), f\left(x_{0}\right), g_{j}\left(t, x_{0}, x_{0}, \varepsilon, \kappa\right) \in \mathcal{R} A_{0}\left(x_{0}\right)$ and other assumptions that will be specified below. Let us remark that (6) is a special kind of the 
general equation (4) with, among other things,

$$
A(x)=\left(\begin{array}{cc}
A_{0}\left(x_{1}\right) & 0 \\
0 & A_{0}\left(x_{2}\right)
\end{array}\right), \quad x=\left(x_{1}, x_{2}\right)
$$

hence $\operatorname{det} A(x)=\operatorname{det} A_{0}\left(x_{1}\right) \operatorname{det} A_{0}\left(x_{2}\right)$ satisfies $\operatorname{det} A\left(x_{0}, x_{0}\right)=0,(\operatorname{det} A)^{\prime}\left(x_{0}, x_{0}\right)=0$ and $(\operatorname{det} A)^{\prime \prime}\left(x_{0}, x_{0}\right) \neq 0$. Thus $\left(x_{0}, x_{0}\right)$ is not a $I$-point. Multiplying the first equation by $A_{0}^{a}\left(x_{1}\right)$ and the second by $A_{0}^{a}\left(x_{2}\right)$ we obtain the system

$$
\begin{aligned}
& \omega\left(x_{1}\right) x_{1}^{\prime}=F\left(x_{1}\right)+\varepsilon G_{1}\left(x_{1}, x_{2}, t, \varepsilon, \kappa\right), \\
& \omega\left(x_{2}\right) x_{2}^{\prime}=F\left(x_{2}\right)+\varepsilon G_{2}\left(x_{1}, x_{2}, t, \varepsilon, \kappa\right) .
\end{aligned}
$$

We assume that $\omega(x):=\operatorname{det} A_{0}(x), F(x)$ and $G_{j}\left(x_{1}, x_{2}, t, \varepsilon, \kappa\right)$ satisfy the following assumptions:

(C1) $F \in C^{2}\left(\mathbb{R}^{2}, \mathbb{R}^{2}\right), \omega \in C^{2}\left(\mathbb{R}^{2}, \mathbb{R}\right)$ and the unperturbed equation

$$
\omega(x) x^{\prime}=F(x)
$$

possesses a noncritical singularity at $x_{0}$, i.e. $\omega\left(x_{0}\right)=0$ and $\omega^{\prime}\left(x_{0}\right) \neq 0$.

(C2) $F\left(x_{0}\right)=0$ and the spectrum $\sigma\left(F^{\prime}\left(x_{0}\right)\right)=\left\{\mu_{ \pm}\right\}$with $\mu_{-}<0<\mu_{+}$, and

$$
x^{\prime}=F(x)
$$

has a solution $\gamma(s)$ homoclinic to $x_{0}$, that is, $\lim _{s \rightarrow \pm \infty} \gamma(s)=x_{0}$, and $\omega(\gamma(s)) \neq 0$ for any $s \in \mathbb{R}$. Without loss of generality, we may, and will, assume $\omega(\gamma(s))>0$ for any $s \in \mathbb{R}$. Moreover, $G_{i} \in C^{2}\left(\mathbb{R}^{6+m}, \mathbb{R}^{2}\right), i=1,2$ are 1-periodic in $t$ with

$G_{i}\left(x_{0}, x_{0}, t, \varepsilon, \kappa\right)=0$ for any $t \in \mathbb{R}, \kappa \in \mathbb{R}^{m}$ and $\varepsilon$ sufficiently small.

(C3) Let $\gamma_{ \pm}$be the eigenvectors of $F^{\prime}\left(x_{0}\right)$ with the eigenvalues $\mu_{\mp}$, resp. Then

$\left\langle\nabla \omega\left(x_{0}\right), \gamma_{ \pm}\right\rangle>0$ (or else $\left.\omega^{\prime}\left(x_{0}\right) \gamma_{ \pm}>0\right)$.

From $(\mathrm{C} 2)$ we see that $\Gamma(s):=\left(\begin{array}{c}\gamma(s) \\ \gamma(s)\end{array}\right)$ is a bounded solution of the equation

$$
\begin{aligned}
& x_{1}^{\prime}=F\left(x_{1}\right), \\
& x_{2}^{\prime}=F\left(x_{2}\right)
\end{aligned}
$$

and that $x_{0}$ persists as a singularity of (7). So this paper is a continuation of $[1,19]$, but here we study more degenerate IODE.

The objective of this paper is to give conditions, besides (C1)-(C3), assuring that for $|\varepsilon| \ll 1$, the coupled equations (7) has a solution in a neighborhood of the orbit $\{\Gamma(s) \mid s \in$ $\mathbb{R}\}$ and reaching $\left(x_{0}, x_{0}\right)$ is a finite time. Our approach mimics that in [1] and uses Melnikov methods to derive the needed conditions. Let us briefly describe the content of this paper. In Section 2 we make few remarks concerning assumptions (C1)-(C3). Then, in Section 3, we change time to reduce equation (7) to a smooth perturbation of (9) whose unperturbed part has the solution $\Gamma(s)$. Next, in Section 4 we derive the Melnikov condition. Finally Section 5 is devoted to the application of our result to coupled equations of the form (1) for RLC circuits, while some computations are postponed to the appendix. 
We emphasize the fact that Melnikov technique is useful to predict the existence of transverse homoclinic orbits in mechanical systems [20,21] together with the associated chaotic behavior of solutions. However, the result in this paper is somewhat different in that we apply the method to show existence of orbits connecting a singularity in finite time.

\section{Comments on the assumptions}

By following [1,19] we note that since $\gamma(s) \rightarrow x_{0}$ as $|s| \rightarrow \infty$ then $\gamma^{\prime}(s)$ is a bounded solution of the linear equation $x^{\prime}=F(\gamma(s)) x$. Hence $\left|\gamma^{\prime}(s)\right| \leq k \mathrm{e}^{-\mu|s|}$ for some $\mu>0$. We get then, for $s \geq 0$,

$$
\left|\gamma(s)-x_{0}\right| \leq \int_{s}^{\infty}\left|\gamma^{\prime}(s)\right| d s \leq \mu^{-1} k \mathrm{e}^{-\mu s} .
$$

So

$$
\limsup _{s \rightarrow \infty} \frac{\log \left|\gamma(s)-x_{0}\right|}{s} \leq-\mu<0
$$

From [22, Theorem 4.3, p.335 and Theorem 4.5, p.338] it follows that

$$
\limsup _{s \rightarrow \infty} \frac{\log \left|\gamma(s)-x_{0}\right|}{s}=\mu_{-}<0 \text {, }
$$

and there exist a constant $\delta>0$ and a solution $\gamma_{+} \mathrm{e}^{\mu_{-} s}$ of $x^{\prime}=F^{\prime}\left(x_{0}\right) x$ such that

$$
\left|\gamma(s)-x_{0}-\gamma_{+} \mathrm{e}^{\mu_{-} s}\right|=O\left(\mathrm{e}^{\left(\mu_{-}-\delta\right) s}\right), \quad \text { as } s \rightarrow \infty .
$$

Note that $\gamma_{+} \neq 0$ since otherwise $\gamma(s)-x_{0}=O\left(\mathrm{e}^{\left(\mu_{-}-\delta\right) s}\right)$, contradicting (10). Hence $\gamma_{+}$is an eigenvector of the eigenvalue $\mu_{-}$of $F^{\prime}\left(x_{0}\right)$. We have then

$$
\left|\frac{\gamma(s)-x_{0}}{\mathrm{e}^{\mu_{-} s}}-\gamma_{+}\right| \leq c_{1} \mathrm{e}^{-\delta s}
$$

for a suitable constant $c_{1} \geq 0$. As a consequence,

$$
\lim _{s \rightarrow \infty} \frac{\gamma(s)-x_{0}}{\mathrm{e}^{\mu_{-} s}}=\gamma_{+} .
$$

Next

$$
\left|\gamma_{+}\right|-c_{1} \mathrm{e}^{-\delta s} \leq \frac{\left|\gamma(s)-x_{0}\right|}{\mathrm{e}^{\mu_{-} s}} \leq\left|\gamma_{+}\right|+c_{1} \mathrm{e}^{-\delta s} .
$$

Taking logarithms, dividing by $s$ and letting $s \rightarrow \infty$ we get

$$
\lim _{s \rightarrow \infty} \frac{\log \left|\gamma(s)-x_{0}\right|}{s}=\mu_{-},
$$

that is, in (10) $\lim \sup _{s \rightarrow \infty}$ can be replaced with $\lim _{s \rightarrow \infty}$. Similarly, changing $s$ with $-s$ :

$$
\lim _{s \rightarrow-\infty} \frac{\log \left|\gamma(s)-x_{0}\right|}{s}=\mu_{+} .
$$


Next, set

$$
\varphi(s):=\frac{1}{\mathrm{e}^{-\mu_{-} s}+\mathrm{e}^{-\mu_{+} s}} .
$$

Since $\frac{\varphi(s)}{\mathrm{e}^{\mu-s}} \rightarrow 1$ as $s \rightarrow \infty$ and $\frac{\varphi(s)}{\mathrm{e}^{\mu+s}} \rightarrow 1$ as $s \rightarrow-\infty$ we have then

$$
\lim _{s \rightarrow \pm \infty} \frac{\gamma(s)-x_{0}}{\varphi(s)}=\gamma_{ \pm} \neq 0
$$

and

$$
\lim _{s \rightarrow \pm \infty} \frac{\omega(\gamma(s))}{\varphi(s)}=\lim _{s \rightarrow \pm \infty} \frac{\omega^{\prime}\left(x_{0}\right)\left(\gamma(s)-x_{0}\right)+o\left(\gamma(s)-x_{0}\right)}{\mathrm{e}^{\mu_{\mp} s}}=\left\langle\nabla \omega\left(x_{0}\right), \gamma_{ \pm}\right\rangle .
$$

From (C2), we know $\omega(\gamma(s))>0$ for any $s \in \mathbb{R}$, so $\left\langle\nabla \omega\left(x_{0}\right), \gamma_{ \pm}\right\rangle \geq 0$. Hence condition (C3) means that $\gamma(s)$ tends transversally to the singular manifold $\omega^{-1}(0)$ at $x_{0}$.

As in [19] it is easily seen that

$$
\lim _{s \rightarrow \pm \infty} \frac{\gamma^{\prime}(s)}{\omega(\gamma(s))}=\frac{F^{\prime}\left(x_{0}\right) \gamma_{ \pm}}{\omega^{\prime}\left(x_{0}\right) \gamma_{ \pm}}=\frac{\mu_{\mp} \gamma_{ \pm}}{\omega^{\prime}\left(x_{0}\right) \gamma_{ \pm}} \neq 0
$$

and that $\frac{\gamma^{\prime}(s)}{\omega(\gamma(s))}$ solves the equation

$$
x^{\prime}=\left[F^{\prime}(\gamma(s))-\frac{F(\gamma(s))}{\omega(\gamma(s))} \omega^{\prime}(\gamma(s))\right] x=F^{\prime}(\gamma(s)) x-\frac{\omega^{\prime}(\gamma(s)) x}{\omega(\gamma(s))} F(\gamma(s)) .
$$

So $\frac{\gamma^{\prime}(s)}{\omega(\gamma(s))}$ is a bounded solution of (14). Next, setting as in [19]

$$
\theta(s):=\int_{0}^{s} \omega(\gamma(\tau)) d \tau
$$

and $x_{h}(t)=\gamma\left(\theta^{-1}(t)\right)$, it is easily seen that $x_{h}(t)$ satisfies $\omega(x) x^{\prime}=F(x)$ whose linearization along $x_{h}(t)$ is

$$
F^{\prime}\left(x_{h}(t)\right) z=x_{h}^{\prime}(t) \omega^{\prime}\left(x_{h}(t)\right) z+\omega\left(x_{h}(t)\right) z^{\prime}=F\left(x_{h}(t)\right) \frac{\omega^{\prime}\left(x_{h}(t)\right) z}{\omega\left(x_{h}(t)\right)}+\omega\left(x_{h}(t)\right) z^{\prime}
$$

i.e.

$$
\omega\left(x_{h}(t)\right) z^{\prime}=F^{\prime}\left(x_{h}(t)\right) z-F\left(x_{h}(t)\right) \frac{\omega^{\prime}\left(x_{h}(t)\right) z}{\omega\left(x_{h}(t)\right)} .
$$

Note, then, that (14) is derived from (16) with the change $x(s)=z(\theta(s))$. This fact should clarify why we need to consider the linear system (14) instead of $x^{\prime}=F^{\prime}(\gamma(s)) x$. However, see [19] for a remark concerning the space of bounded solutions of (14) and that of the equation $x^{\prime}=F^{\prime}(\gamma(s)) x$.

We now prove that $\frac{\gamma^{\prime}(s)}{\omega(\gamma(s))}$ is the unique solution of equation (14) which is bounded on $\mathbb{R}$. This is a kind of nondegeneracy of $\gamma(s)$. 
Lemma 2.1 Assume (C2) and (C3) hold. Then, up to a multiplicative constant, $\frac{\gamma^{\prime}(s)}{\omega(\gamma(s))}$ is the unique solution of (14) which is bounded on $\mathbb{R}$.

Proof From [19, Lemma 3.1] it follows that the linear map:

$$
x \mapsto\left[F^{\prime}\left(x_{0}\right)-\frac{\mu_{-} \gamma_{+}}{\omega^{\prime}\left(x_{0}\right) \gamma_{+}} \omega^{\prime}\left(x_{0}\right)-\mu_{-} \mathbb{I}\right] x
$$

has the simple eigenvalues $\mu_{+}-\mu_{-}$and $-\mu_{-}$. Let $\mu:=\frac{\mu_{+}}{2}$, then the linear map

$$
x \mapsto\left[F^{\prime}\left(x_{0}\right)-\frac{\mu_{-} \gamma_{+}}{\omega^{\prime}\left(x_{0}\right) \gamma_{+}} \omega^{\prime}\left(x_{0}\right)-\mu \mathbb{I}\right] x
$$

has the eigenvalues $\pm \mu$; moreover, since

$$
c_{1} \leq \frac{\left|\gamma^{\prime}(s)\right|}{\omega(\gamma(s))} \leq c_{2}
$$

for two positive constants $0<c_{1}<c_{2}$, it follows that $\gamma_{0}(s):=\frac{\gamma^{\prime}(s)}{\omega(\gamma(s))} \mathrm{e}^{-\mu s}$ is a solution of

$$
x^{\prime}=\left[F^{\prime}(\gamma(s))-\frac{F(\gamma(s))}{\omega(\gamma(s))} \omega^{\prime}(\gamma(s))-\mu \mathbb{I}\right] x
$$

satisfying

$$
\frac{c_{1}}{c_{2}}\left|\gamma_{0}\left(s_{1}\right)\right| \leq\left|\gamma_{0}\left(s_{2}\right)\right| \mathrm{e}^{\mu\left(s_{2}-s_{1}\right)} \leq \frac{c_{2}}{c_{1}}\left|\gamma_{0}\left(s_{1}\right)\right|
$$

for all $0 \leq s_{1} \leq s_{2}$. Then (17) satisfies the assumptions of [19, Theorem 5.3] and hence its conclusion with $\operatorname{rank} P_{+}=1$, that is, the fundamental matrix $X_{+}(s)$ of (17) satisfies

$$
\begin{aligned}
& \left\|X_{+}\left(s_{2}\right) P_{+} X_{+}^{-1}\left(s_{1}\right)\right\| \leq k \mathrm{e}^{-\mu\left(s_{2}-s_{1}\right)}, \quad 0 \leq s_{1} \leq s_{2}, \\
& \left\|X_{+}\left(s_{2}\right)\left(\mathbb{I}-P_{+}\right) X_{+}^{-1}\left(s_{1}\right)\right\| \leq k \mathrm{e}^{\tilde{\mu}\left(s_{2}-s_{1}\right)}, \quad 0 \leq s_{2} \leq s_{1},
\end{aligned}
$$

where $0 \leq \tilde{\mu}<\mu$. However, it is well known (see [23-25]) that $\mathcal{R} P_{+}$is the space of initial conditions for the bounded solutions on [0, $\infty$ [ of (17) that, then, tend to zero as $s \rightarrow \infty$ at the exponential rate $\mathrm{e}^{-\mu s}$. As a consequence a solution $u(s)$ of (17) is bounded on $[0, \infty[$ if and only if $u(s) \mathrm{e}^{\mu s}$ is a bounded solution of (14). Then we conclude that the space of solutions of (14) that are bounded on $[0, \infty[$ is one dimensional.

Incidentally, since the fundamental matrix of (14) is $X(s)=X_{+}(s) \mathrm{e}^{\mu s}$, we note that it satisfies

$$
\begin{aligned}
& \left\|X\left(s_{2}\right) P_{+} X^{-1}\left(s_{1}\right)\right\| \leq k, \quad 0 \leq s_{1} \leq s_{2}, \\
& \left\|X\left(s_{2}\right)\left(\mathbb{I}-P_{+}\right) X^{-1}\left(s_{1}\right)\right\| \leq k \mathrm{e}^{(\mu+\tilde{\mu})\left(s_{2}-s_{1}\right)}, \quad 0 \leq s_{2} \leq s_{1} .
\end{aligned}
$$

Using a similar argument in $\left.\left.\mathbb{R}_{-}=\right]-\infty, 0\right]$ with $\mu=\frac{\mu_{-}}{2}<0$, and [19, Theorem 5.4] instead of [19, Theorem 5.3] with $\mu^{*}=-\mu$ we see that (14) has at most a one dimensional space 
of solutions bounded in $\mathbb{R}$. More precisely, $\tilde{\mu}$ with $\mu<\tilde{\mu}<0$, and a projection $P_{-}$on $\mathbb{R}^{2}$ exists such that

$$
\begin{aligned}
& \left\|X\left(s_{2}\right)\left(\mathbb{I}-P_{-}\right) X^{-1}\left(s_{1}\right)\right\| \leq k, \quad s_{2} \leq s_{1} \leq 0, \\
& \left\|X\left(s_{2}\right) P_{-} X^{-1}\left(s_{1}\right)\right\| \leq k \mathrm{e}^{(\mu+\tilde{\mu})\left(s_{2}-s_{1}\right)}, \quad s_{1} \leq s_{2} \leq 0,
\end{aligned}
$$

and $\operatorname{dim} \mathcal{N} P_{-}=1$. Since $\frac{\gamma^{\prime}(s)}{\omega(\gamma(s))}$ is a solution of (14) bounded on $\mathbb{R}$ we deduce that $\mathcal{R} P_{+}=$ $\mathcal{N} P_{-}=\operatorname{span}\left\{\frac{\gamma^{\prime}(0)}{\omega(\gamma(0))}\right\}$ and the result follows.

We conclude this section with a remark about condition (c) in [19, Theorem 5.3]. Consider a system in $\mathbb{R}^{n}$ such as

$$
x^{\prime}=[D+A(s)] x .
$$

Then the following result holds.

\section{Theorem 2.2 Suppose the following hold:}

(i) D has two simple eigenvalues $\mu_{*}<\mu^{*}$ and all the other eigenvalues of $D$ have either real part less than $\mu_{*}$ or greater than $\mu^{*}$;

(ii) $\int_{0}^{\infty}\|A(s)\| d s<\infty$;

(iii) $A(s) \rightarrow 0$ as $s \rightarrow \infty$.

Then there are as many solutions $x(t)$ of (18) satisfying

$$
k_{1}|x(s)| \leq|x(t)| \mathrm{e}^{-\mu_{*}(t-s)} \leq k_{2}|x(s)|, \quad \text { for any } 0 \leq s \leq t,
$$

as the dimension of the space of the generalized eigenvectors of the matrix $D$ with real parts less than or equal to $\mu_{*}$; here $k_{1}, k_{2}>0$ are two suitable positive constants. Similarly there are as many solutions of (18) such that

$$
\tilde{k}_{1}|x(s)| \leq|x(t)| \mathrm{e}^{-\mu^{*}(t-s)} \leq \tilde{k}_{2}|x(s)|, \quad \text { for any } 0 \leq s \leq t,
$$

for suitable constants $\tilde{k}_{1}, \tilde{k}_{2}>0$, as the dimension of the space of the generalized eigenvectors of the matrix $D$ with real parts greater than or equal to $\mu^{*}$.

Proof We prove the first statement concerning (19). By a similar argument (20) is handled. Changing variables we may assume that

$$
D=\left(\begin{array}{ccc}
\mu_{*} & 0 & 0 \\
0 & D_{-} & 0 \\
0 & 0 & D_{+}
\end{array}\right)
$$

and the eigenvalues of $D_{-}$have real parts less than $\mu_{*}$ and those of $D_{+}$have real parts greater than or equal to $\mu^{*}$. So the system reads

$$
\begin{aligned}
& x_{1}^{\prime}=\mu_{*} x_{1}+a_{11}(t) x_{1}+A_{12}(t) x_{2}+A_{13}(t) x_{3}, \\
& x_{2}^{\prime}=D_{-} x_{2}+A_{21}(t) x_{1}+A_{22}(t) x_{2}+A_{23}(t) x_{3}, \\
& x_{3}^{\prime}=D_{+} x_{3}+A_{31}(t) x_{1}+A_{32}(t) x_{2}+A_{33}(t) x_{3},
\end{aligned}
$$


where $a_{11}(t) \in \mathbb{R}$ and $A_{i j}(t)$ are matrices (or vectors) of suitable orders. Setting $y_{i}(t)=$ $\mathrm{e}^{-\mu_{*} t} x_{i}(t)$ we get

$$
\begin{aligned}
& y_{1}^{\prime}=a_{11}(t) y_{1}+A_{12}(t) y_{2}+A_{13}(t) y_{3}, \\
& y_{2}^{\prime}=\left(D_{-}-\mu_{*} \mathbb{I}\right) y_{2}+A_{21}(t) y_{1}+A_{22}(t) y_{2}+A_{23}(t) y_{3}, \\
& y_{3}^{\prime}=\left(D_{+}-\mu_{*} \mathbb{I}\right) y_{3}+A_{31}(t) y_{1}+A_{32}(t) y_{2}+A_{33}(t) y_{3} .
\end{aligned}
$$

Now we observe that $y(t)$ is a solution of (22) bounded at $+\infty$ if and only if $x(t)$ is a solution of (21) which is bounded on $\mathbb{R}$ when multiplied by $\mathrm{e}^{-\mu_{* t}}$. Moreover, since $\left|a_{11}(t)\right|,\left|A_{12}(t)\right|$, and $\left|A_{13}(t)\right|$ belong to $L^{1}(\mathbb{R})$, the $\operatorname{limit}_{t \rightarrow+\infty} y_{1}(t)$ exists for any solution $y(t)$ of $(22)$ bounded on $\mathbb{R}_{+}$. So, let us fix $t_{0}>0$ and take $t \geq t_{0}$. If $y(t)$ is a solution of (22) bounded at $+\infty$ it must be, by the variation of constants formula,

$$
\begin{aligned}
y_{1}(t)= & y_{1}^{\infty}-\int_{t}^{\infty}\left(a_{11}(s) y_{1}(s)+A_{12}(s) y_{2}(s)+A_{13}(s) y_{3}(s)\right) d s, \\
y_{2}(t)= & \mathrm{e}^{\left(D_{-}-\mu_{*} \mathbb{I}\right)\left(t-t_{0}\right)} y_{2}^{0} \\
& +\int_{t_{0}}^{t} \mathrm{e}^{\left(D_{-}-\mu_{*} \mathbb{I}\right)(t-s)}\left(A_{21}(s) y_{1}(s)+A_{22}(s) y_{2}(s)+A_{23}(s) y_{3}(s)\right) d s, \\
y_{3}(t)= & -\int_{t}^{\infty} \mathrm{e}^{\left(D_{+}-\mu_{*} \mathbb{I}\right)(t-s)}\left(A_{31}(s) y_{1}(s)+A_{32}(s) y_{2}(s)+A_{33}(s) y_{3}(s)\right) d s,
\end{aligned}
$$

where $y_{2}^{0}=y_{2}\left(t_{0}\right)$ and $y_{1}^{\infty}=\lim _{t \rightarrow+\infty} y_{1}(t)$. Note that since $\sigma\left(D_{-}-\mu_{*} \mathbb{I}\right) \subset\{\lambda \in \mathbb{C} \mid \Re \lambda<0\}$ and $\sigma\left(D_{+}-\mu_{*} \mathbb{I}\right) \subset\{\lambda \in \mathbb{C} \mid \Re \lambda>0\}$ and $a_{11}(t), A_{i j}(t)$ are bounded, we can interpret (22) as a fixed point theorem in the Banach space of bounded function on $\left[t_{0},+\infty[\right.$ :

$$
B:=\left\{y ( t ) \in C ^ { 0 } \left(\left[t_{0}, \infty[)|\sup | y(t) \mid<\infty\right\}\right.\right.
$$

with the obvious norm. Since $a_{11}(t), A_{i j}(t) \in L^{1}\left(\mathbb{R}_{+}\right)$we see that the map (23) is a contraction on $B$, provided $t_{0}$ is sufficiently large, and then, for any given $\left(y_{1}^{\infty}, y_{2}^{0}\right)$, it has a unique solution $y\left(t, y_{1}^{\infty}, y_{2}^{0}\right) \in B$. Note that a priori $y\left(t, y_{1}^{\infty}, y_{2}^{0}\right)$ is defined only on $\left[t_{0},+\infty\right.$ [ but of course we can extend it to $[0,+\infty$ [ going backward with time. We now prove that positive constants $0<c_{1}<c_{2}$ exist such that $c_{1} \leq\left|y\left(t, y_{0}\right)\right| \leq c_{2}$ fox any $t \geq 0$. Let $t_{0}<t_{1}<t$. We have

$$
\begin{aligned}
y_{2}\left(t_{1}\right)= & \mathrm{e}^{\left(D_{-}-\mu_{*} \mathbb{I}\right)\left(t_{1}-t_{0}\right)} y_{2}^{0} \\
& +\int_{t_{0}}^{t_{1}} \mathrm{e}^{\left(D_{-}-\mu_{*} \mathbb{I}\right)\left(t_{1}-s\right)}\left(A_{21}(s) y_{1}(s)+A_{22}(s) y_{2}(s)+A_{23}(s) y_{3}(s)\right) d s
\end{aligned}
$$

and then

$$
\begin{aligned}
y_{2}(t)= & \mathrm{e}^{\left(D_{-}-\mu_{*} \mathbb{I}\right)\left(t-t_{0}\right)} y_{2}^{0}+\int_{t_{0}}^{t_{1}} \mathrm{e}^{\left(D_{-}-\mu_{*} \mathbb{I}\right)(t-s)}\left(A_{21}(s) y_{1}(s)+A_{22}(s) y_{2}(s)+A_{23}(s) y_{3}(s)\right) d s \\
& +\int_{t_{1}}^{t} \mathrm{e}^{\left(D_{-}-\mu_{*} \mathbb{I}\right)(t-s)}\left(A_{21}(s) y_{1}(s)+A_{22}(s) y_{2}(s)+A_{23}(s) y_{3}(s)\right) d s \\
= & \mathrm{e}^{\left(D_{-}-\mu_{*} \mathbb{I}\right)\left(t-t_{1}\right)} y_{2}\left(t_{1}\right)+\int_{t_{1}}^{t} \mathrm{e}^{\left(D_{-}-\mu_{*} \mathbb{I}\right)(t-s)}\left(A_{21}(s) y_{1}(s)+A_{22}(s) y_{2}(s)+A_{23}(s) y_{3}(s)\right) d s .
\end{aligned}
$$


So, for any $\delta>0$ let $t_{1}$ be such that $\sup _{t \geq t_{1}}\left|A_{i j}(t)\right| \leq \delta$ and set $\sup _{t \geq t_{1}}\left|y_{j}(t)\right|=\bar{y}_{j}$. We have

$$
\begin{aligned}
\bar{y}_{2} & =\sup _{t \geq t_{1}}\left|y_{2}(t)\right| \leq k \mathrm{e}^{\alpha\left(t-t_{1}\right)} \bar{y}_{2}+\int_{t_{1}}^{t} k \mathrm{e}^{\alpha(t-s)} \delta\left(\bar{y}_{1}+\bar{y}_{2}+\bar{y}_{3}\right) d s \\
& \leq k\left(\bar{y}_{2}+\frac{\delta}{\alpha}\left(\bar{y}_{1}+\bar{y}_{2}+\bar{y}_{3}\right)\right) \mathrm{e}^{\alpha\left(t-t_{1}\right)}+\frac{k \delta}{|\alpha|}\left(\bar{y}_{1}+\bar{y}_{2}+\bar{y}_{3}\right)
\end{aligned}
$$

with $\max \left\{\Re \mu \mid \mu \in \sigma\left(D_{-}-\mu_{*} \mathbb{I}\right)\right\}<\alpha<0$. Taking the limit as $t \rightarrow+\infty$ we get

$$
\bar{y}_{2} \leq \frac{\delta}{|\alpha|}\left(\bar{y}_{1}+\bar{y}_{2}+\bar{y}_{3}\right)
$$

Since $\delta \rightarrow 0$ as $t_{1} \rightarrow+\infty$, from the above it follows that $\lim _{t \rightarrow \infty}\left|y_{2}(t)\right|=0$. Similarly we get

$$
\left|y_{3}\right| \leq k \delta \beta^{-1}\left(\bar{y}_{1}+\bar{y}_{2}+\bar{y}_{3}\right)
$$

where $0<\beta<\min \left\{\Re \mu \mid \mu \in \sigma\left(D_{+}-\mu_{*} \mathbb{I}\right)\right\}$ and then $\lim _{t \rightarrow \infty}\left|y_{3}(t)\right|=0$. As a consequence we obtain $\lim _{t \rightarrow \infty}|y(t)|-\left|y_{1}(t)\right|=0$ and then

$$
\lim _{t \rightarrow \infty}|y(t)|=\left|y_{1}^{\infty}\right|
$$

So, provided we take $y_{1}^{\infty} \neq 0$ we see that eventually (i.e. for $t \geq \bar{t}$, for some $\bar{t}>0$ )

$$
\frac{\left|y_{1}^{\infty}\right|}{2} \leq|y(t)| \leq \frac{3}{2}\left|y_{1}^{\infty}\right|
$$

and the existence of $c_{1}, c_{2}>0$ such that

$$
c_{1} \leq|y(t)| \leq c_{2}
$$

for all $t \geq 0$ follows from the fact that $|y(t)|$ cannot vanish in any bounded interval. Finally since $|x(t)|=|y(t)| \mathrm{e}^{\mu_{*} t}$ we get, for $0 \leq s \leq t$,

$$
\frac{|x(t)|}{|x(s)|}=\frac{|y(t)|}{|y(s)|} \mathrm{e}^{\mu_{*}(t-s)} \Rightarrow \frac{c_{1}}{c_{2}} \mathrm{e}^{\mu_{*}(t-s)} \leq \frac{|x(t)|}{|x(s)|} \leq \frac{c_{2}}{c_{1}} \mathrm{e}^{\mu_{*}(t-s)}
$$

i.e.

$$
\frac{c_{1}}{c_{2}}|x(s)| \leq|x(t)| \mathrm{e}^{-\mu_{*}(t-s)} \leq \frac{c_{2}}{c_{1}}|x(s)| .
$$

The proof is complete.

Remark 2.3 (i) It follows from the proof of Theorem 2.2 that inequalities of (19) also hold replacing (i) with the weaker assumption that $\mu_{*}$ is a simple eigenvalue of $D$ and all the others either have real parts less than $\mu_{*}$ or $\geq \mu^{*}$ (i.e. we do not need that $\mu^{*}$ is simple). Similarly inequalities of (20) hold if $\mu^{*}$ is a simple eigenvalue of $D$ and all the others either have real parts greater than $\mu^{*}$ or $\leq \mu_{*}$ (i.e. we do not need that $\mu_{*}$ is simple).

(ii) Note that a result related to Theorem 2.2 has been proved in [26]. 


\section{Solutions asymptotic to the fixed point}

It follows from (11)-(12) that $\gamma(s)-x_{0}=O\left(\mathrm{e}^{\mu_{\mp} s}\right)$ as $s \rightarrow \pm \infty$ then, since $\gamma^{\prime}(s)=F(\gamma(s))=$ $O\left(\gamma(s)-x_{0}\right)$ we obtain $\gamma^{\prime}(s)=O\left(\mathrm{e}^{\mu_{\mp} s}\right)$. Furthermore, from (13) we also get:

$$
\omega(\gamma(s))=O\left(\gamma^{\prime}(s)\right)=O\left(\mathrm{e}^{\mu_{\mp} s}\right)
$$

As a consequence

$$
T_{ \pm}:=\int_{0}^{ \pm \infty} \omega(\gamma(\tau)) d \tau<\infty
$$

Since $\omega(\gamma(s))>0$ it follows that $\theta: \mathbb{R} \rightarrow] T_{-}, T_{+}[$is a strictly increasing diffeomorphism (see (15) for the definition of $\theta(s))$. Then $x_{h}(t):=\gamma\left(\theta^{-1}(t)\right)$ satisfies (8) on the interval ]$T_{-}, T_{+}[$and

$$
\lim _{t \rightarrow T_{ \pm}} x_{h}(t)=x_{0}
$$

Moreover (see (13))

$$
\lim _{t \rightarrow t_{ \pm}} x_{h}^{\prime}(t)=\lim _{t \rightarrow t_{ \pm}} \frac{F\left(x_{h}(t)\right)}{\omega\left(x_{h}(t)\right)}=\lim _{s \rightarrow \pm \infty} \frac{F(\gamma(s))}{\omega(\gamma(s))}=\mu_{\mp} \frac{\gamma_{ \pm}}{\omega^{\prime}\left(x_{0}\right) \gamma_{ \pm}} \neq 0 .
$$

Hence $x_{0}$ is not an $I$-point of (8). In this paper we want to look for solutions of the coupled equation (7) that belong to a neighborhood of $\left\{\left(x_{h}(t), x_{h}(t)\right) \mid T_{-}<t<T_{+}\right\}$, they are defined in the interval $] T_{-}+\alpha, T_{+}+\alpha$ [, for some $\alpha=\alpha(\varepsilon)$, and tend to $\left(x_{0}, x_{0}\right)$ at the same rate as $\left(x_{h}(t), x_{h}(t)\right)$. To this end we first perform a change of the time variable as follows. Set

$$
t=\alpha+\theta(s) \in] T_{-}+\alpha, T_{+}+\alpha[
$$

and plug $z_{j}(s)=x_{j}(\alpha+\theta(s))$ in (7). We get

$$
\omega\left(z_{j}\right) z_{j}^{\prime}=\omega(\gamma(s))\left(F\left(z_{j}\right)+\varepsilon G_{j}\left(z_{1}, z_{2}, \alpha+\theta(s), \varepsilon, \kappa\right)\right), \quad j=1,2 .
$$

Since we are looking for solutions of (7) tending to $\left(x_{0}, x_{0}\right)$ at the same rate as $\gamma(s)$, in (24) we make the change of variables

$$
z_{j}(s)=\gamma(s)+\varphi(s) y_{j}(s)=x_{0}+\varphi(s)\left(\eta(s)+y_{j}(s)\right), \quad j=1,2,
$$

where $\eta(s)$ is the bounded function $\frac{\gamma(s)-x_{0}}{\varphi(s)}$. Since

$$
\begin{aligned}
& \omega\left(x_{0}+\varphi(s)(\eta(s)+y)\right) \\
& \quad \geq\left\langle\nabla \omega\left(x_{0}\right), \varphi(s)(\eta(s)+y)\right\rangle-K_{1}|\varphi(s)(\eta(s)+y)|^{2} \\
& \quad=\varphi(s)\left[\left\langle\nabla \omega\left(x_{0}\right), \eta(s)+y\right\rangle-K_{1} \varphi(s)|\eta(s)+y|^{2}\right]
\end{aligned}
$$

for a suitable constant $K_{1}>0$ and any $s \in \mathbb{R},|y| \leq 1$ we get, using (C3), (26):

$$
\omega\left(x_{0}+\varphi(s)(\eta(s)+y)\right) \geq \frac{1}{2} \varphi(s)\left\langle\nabla \omega\left(x_{0}\right), \gamma_{ \pm}\right\rangle>0
$$


for $|s|>0$ large and $|y|<\delta$ sufficiently small. Then (27) and $\omega(\gamma(t))>0$ imply the existence of $M>0$ and $\delta>0$ so that

$$
\omega\left(x_{0}+\varphi(s)(\eta(s)+y)\right) \geq M \varphi(s)
$$

for any $s \in \mathbb{R}$ and $|y| \leq \delta$. Now plugging (25) into (24) we derive the equations

$$
\begin{aligned}
y_{j}^{\prime}= & \frac{\omega(\gamma(s))}{\varphi(s) \omega\left(\gamma(s)+\varphi(s) y_{j}\right)} F\left(\gamma(s)+\varphi(s) y_{j}\right)-\frac{F(\gamma(s))}{\varphi(s)}-\frac{\varphi^{\prime}(s)}{\varphi(s)} y_{j} \\
& +\varepsilon \frac{\omega(\gamma(s))}{\varphi(s) \omega\left(\gamma(s)+\varphi(s) y_{j}\right)} G_{j}\left(\gamma(s)+\varphi(s) y_{1}, \gamma(s)+\varphi(s) y_{2}, \theta(s)+\alpha, \varepsilon, \kappa\right), \\
& j=1,2 .
\end{aligned}
$$

From (11) it follows that

$$
\frac{\varphi^{\prime}(s)}{\varphi(s)}=\frac{\mu_{-} \mathrm{e}^{-\mu_{-}(s)}+\mu_{+} \mathrm{e}^{-\mu_{+}(s)}}{\mathrm{e}^{-\mu_{-}(s)}+\mathrm{e}^{-\mu_{+}(s)}} \rightarrow \mu_{\mp} \quad \text { as } s \rightarrow \pm \infty .
$$

Next we note that from $G_{j}\left(x_{0}, x_{0}, t, \varepsilon, \kappa\right)=0$ it follows that the quantities

$$
\begin{aligned}
& \frac{G_{j}\left(\gamma(s)+\varphi(s) y_{1}, \gamma(s)+\varphi(s) y_{2}, \alpha+\theta(s), \varepsilon, \kappa\right)}{\varphi(s)} \\
& \quad=\frac{G_{j}\left(x_{0}+\varphi(s)\left(\eta(s)+y_{1}\right), x_{0}+\varphi(s)\left(\eta(s)+y_{2}\right), \alpha+\theta(s), \varepsilon, \kappa\right)}{\varphi(s)}, \quad j=1,2
\end{aligned}
$$

are bounded uniformly in $s \in \mathbb{R}$ and $\kappa \in \mathbb{R}^{m}, y_{1}, y_{2}$, $\varepsilon$ bounded.

The linearization of (28) at $y=0, \varepsilon=0$ is

$$
y_{j}^{\prime}=\left[F^{\prime}(\gamma(s))-\frac{F(\gamma(s)) \omega^{\prime}(\gamma(s))}{\omega(\gamma(s))}-\frac{\varphi^{\prime}(s)}{\varphi(s)} \mathbb{I}\right] y_{j}, \quad j=1,2 .
$$

Taking the limit as $s \rightarrow+\infty$ we get the systems

$$
y_{j}^{\prime}=\left[F^{\prime}\left(x_{0}\right)-\frac{\mu_{-} \gamma_{+}}{\omega^{\prime}\left(x_{0}\right) \gamma_{+}} \omega^{\prime}\left(x_{0}\right)-\mu_{-} \mathbb{I}\right] y_{j}, \quad j=1,2 .
$$

Similarly taking the limit as $s \rightarrow-\infty$ we get the systems

$$
y_{j}^{\prime}=\left[F^{\prime}\left(x_{0}\right)-\frac{\mu_{+} \gamma_{-}}{\omega^{\prime}\left(x_{0}\right) \gamma_{-}} \omega^{\prime}\left(x_{0}\right)-\mu_{+} \mathbb{I}\right] y_{j}, \quad j=1,2 .
$$

From the proof of Lemma 2.1 (see also [1, Lemma 3.1]) we know that (30) has the positive simple eigenvalues $\mu_{+}-\mu_{-}$and $-\mu_{-}$, and (31) has the negative simple eigenvalues $\mu_{-}-\mu_{+}$ and $-\mu_{+}$. From the roughness of exponential dichotomies it follows that both equations in (29) have an exponential dichotomy on both $\mathbb{R}_{+}$and $\mathbb{R}_{-}$with projections, resp. $P_{+}=0$ and $P_{-}=\mathbb{I}$. Hence (see also [19]) all solutions of the system

$$
y^{\prime}=-\left[F^{\prime}(\gamma(s))^{*}-\frac{\omega^{\prime}(\gamma(s))^{*} F(\gamma(s))^{*}}{\omega(\gamma(s))}-\frac{\varphi^{\prime}(s)}{\varphi(s)} \mathbb{I}\right] y,
$$


adjoint to (29), are bounded as $|s| \rightarrow \infty$. We let $\psi_{1}(s)$ and $\psi_{2}(s)$ be any two linearly independent solutions of (32).

\section{Melnikov function and the original equation}

In this section we will give a condition for solving (28) for $y_{1}(t), y_{2}(t)$ near the solution $y_{1}(t)=y_{2}(t)=0$ of the same equation with $\varepsilon=0$. Writing

$$
\mathcal{F}(y):=y^{\prime}-\frac{\omega(\gamma(s))}{\varphi(s) \omega(\gamma(s)+\varphi(s) y)} F(\gamma(s)+\varphi(s) y)+\frac{F(\gamma(s))}{\varphi(s)}+\frac{\varphi^{\prime}(s)}{\varphi(s)} y
$$

and

$$
\begin{aligned}
H_{j}\left(y_{1}, y_{2}, \eta, \varepsilon\right):= & -\frac{\omega(\gamma(s))}{\varphi(s) \omega\left(\gamma(s)+\varphi(s) y_{j}\right)} \\
& \times G_{j}\left(\gamma(s)+\varphi(s) y_{1}, \gamma(s)+\varphi(s) y_{2}, \theta(s)+\alpha, \varepsilon, \kappa\right), \quad j=1,2,
\end{aligned}
$$

we look for solutions $y_{1}(t), y_{2}(t): \mathbb{R} \rightarrow \mathbb{R}^{2}$ of

$$
\begin{aligned}
& \mathcal{F}\left(y_{1}\right)+\varepsilon H_{1}\left(y_{1}, y_{2}, \eta, \varepsilon\right)=0, \\
& \mathcal{F}\left(y_{2}\right)+\varepsilon H_{2}\left(y_{1}, y_{2}, \eta, \varepsilon\right)=0
\end{aligned}
$$

in the Banach space of $C^{1}$-functions on $\mathbb{R}$, bounded together with their derivatives and with small norms. We observe that $\mathcal{F}(0)=0$ and equation $\mathcal{F}^{\prime}(0) y=0$ reads

$$
y^{\prime}=\left[F^{\prime}(\gamma(s))-\frac{F(\gamma(s)) \omega^{\prime}(\gamma(s))}{\omega(\gamma(s))}-\frac{\varphi^{\prime}(s)}{\varphi(s)}\right] y
$$

In Section 3 (see also $[1,19]$ ) we have seen that (36) has an exponential dichotomy on both $\mathbb{R}_{+}$and $\mathbb{R}_{+}$with projections $P_{+}=\mathbb{I}-P_{-}=0$. So the only bounded solution $y(t)$ of $\mathcal{F}^{\prime}(0) y=0$ is $y(t)=0$. In other words $\mathcal{N} \mathcal{F}^{\prime}(0)=\{0\}$. So we are lead to prove the following.

Theorem 4.1 Let $Y, X$ be Banach spaces, $\varepsilon \in \mathbb{R}$ be a small parameter and $\eta \in \mathbb{R}^{2 d}$. Let $\mathcal{F}: Y \rightarrow X, H_{1,2}: Y \times Y \times \mathbb{R}^{2 d+1} \rightarrow X,\left(y_{1}, y_{2}, \eta, \varepsilon\right) \mapsto H_{1,2}\left(y_{1}, y_{2}, \eta, \varepsilon\right)$ be $C^{2}$-functions such that

(a) $\mathcal{F}(0)=0$;

(b) $\mathcal{N F}^{\prime}(0)=\{0\}$;

(c) there exist $\psi_{1}, \ldots, \psi_{d} \in X^{*}$ such that $\mathcal{R F}^{\prime}(0)=\left\{\psi_{1}, \ldots, \psi_{d}\right\}^{\circ}$.

Set $\mathcal{M}: \mathbb{R}^{2 d} \rightarrow \mathbb{R}^{2 d}$ by

$$
\mathcal{M}(\eta):=\left(\begin{array}{c}
\psi_{1} H_{1}(0,0,0, \eta, 0) \\
\vdots \\
\psi_{d} H_{1}(0,0,0, \eta, 0) \\
\psi_{1} H_{2}(0,0,0, \eta, 0) \\
\vdots \\
\psi_{d} H_{2}(0,0,0, \eta, 0)
\end{array}\right)
$$


and suppose there exists $\bar{\eta} \in \mathbb{R}^{2 d}$ such that $\mathcal{M}(\bar{\eta})=0$ and the derivative $\mathcal{M}^{\prime}(\bar{\eta})$ is invertible. Then there exist $r>0$ and unique $C^{1}$-function $\eta=\eta(\varepsilon)$, defined in a neighborhood of $\varepsilon=$ $0 \in \mathbb{R}$ such that

$$
\lim _{\varepsilon \rightarrow 0} \eta(\varepsilon)=\bar{\eta}
$$

and for $\eta=\eta(\varepsilon), \varepsilon \neq 0$, (35) has a unique solution $\left(y_{1}(\varepsilon), y_{2}(\varepsilon)\right) \in Y \times Y$ satisfying

$$
\left\|\left(y_{1}(\varepsilon), y_{2}(\varepsilon)\right)\right\| \leq r .
$$

Moreover, $y_{j}(\varepsilon)=\varepsilon \widetilde{y}_{j}(\varepsilon)$ for $C^{0}$-functions $\tilde{y}_{j}(\varepsilon) \in Y$ and we have

$$
\mathcal{F}^{\prime}(0) \widetilde{y}_{j}(0)+H_{j}(0,0,0, \bar{\eta}, 0)=0, \quad j=1,2 .
$$

Proof We look for solutions $\left(y_{1}, y_{2}, \eta\right)$ of $(35)$ that are close to $\left(y_{1}, y_{2}, \eta\right)=(0,0, \bar{\eta})$. Let $P: X \rightarrow X$ be the projection such that $\mathcal{R} P=\mathcal{R F}^{\prime}(0)$. Note $\operatorname{codim} \mathcal{R} \mathcal{F}^{\prime}(0)=d$. From the implicit function theorem, we solve the projected equations

$$
\begin{aligned}
& P \mathcal{F}\left(y_{1}\right)+\varepsilon P H_{1}\left(y_{1}, y_{2}, \eta, \varepsilon\right)=0, \\
& P \mathcal{F}\left(y_{2}\right)+\varepsilon P H_{2}\left(y_{1}, y_{2}, \eta, \varepsilon\right)=0
\end{aligned}
$$

for unique $y_{1,2}=Y_{1,2}(\eta, \varepsilon) \in Y$ such that

$$
Y_{1,2}(\eta, 0)=0
$$

provided $|\varepsilon| \leq \varepsilon_{0}$ is sufficiently small and $\eta$ in a fixed closed ball $\Xi \subset \mathbb{R}^{2 d}$ with $\bar{\eta} \in \stackrel{\circ}{\Xi}$. Note that $Y_{1,2}$ are $C^{2}$-smooth. Setting $Q=\mathbb{I}-P$, we need to solve the bifurcation equations:

$$
Q \mathcal{F}\left(Y_{j}(\eta, \varepsilon)\right)+\varepsilon Q H_{j}\left(Y_{1}(\eta, \varepsilon), Y_{2}(\eta, \varepsilon), \eta, \varepsilon\right)=0, \quad j=1,2 .
$$

Observe that

$$
Q x=0 \quad \Leftrightarrow \quad x \in \mathcal{R} P=\mathcal{R F}^{\prime}(0) \quad \Leftrightarrow \quad \psi_{i} x=0 \quad \text { for all } i=1, \ldots, d .
$$

Then $Q \mathcal{F}^{\prime}(0)=0$ and so

$$
Q \mathcal{F}\left(Y_{j}(\eta, \varepsilon)\right)=O_{j}\left(\varepsilon^{2}\right), \quad j=1,2,
$$

uniformly with respect to $\eta$. We conclude that (40) can be written as

$$
\varepsilon Q H_{j}(0,0, \eta, 0)=-Q R_{j}(\eta, \varepsilon), \quad j=1,2,
$$

where

$$
R_{j}(\eta, \varepsilon):=\mathcal{F}\left(Y_{j}(\eta, \varepsilon)\right)+\varepsilon\left[H_{j}\left(Y_{1}(\eta, \varepsilon), Y_{2}(\eta, \varepsilon), \eta, \varepsilon\right)-H_{j}(0,0, \eta, 0)\right] .
$$


Note that $R_{j}(\eta, \varepsilon)$ are $C^{2}$-functions of $(\eta, \varepsilon)$ and that $\varepsilon^{-1} R_{j}(\eta, \varepsilon)=O(\varepsilon)$ uniformly with respect to $\eta$, so

$$
\widetilde{R}_{j}(\eta, \varepsilon):= \begin{cases}-\varepsilon^{-1} R_{j}(\eta, \varepsilon), & \text { if } \varepsilon \neq 0, \\ 0, & \text { if } \varepsilon=0\end{cases}
$$

is $C^{1}$ in $(\eta, \varepsilon)$. By (41), system (42) is equivalent to

$$
\mathcal{M}(\eta)=\left(\begin{array}{l}
\psi_{i} \widetilde{R}_{1}(\eta, \varepsilon) \\
\psi_{i} \widetilde{R}_{2}(\eta, \varepsilon)
\end{array}\right)_{i=1, \ldots, d}=O(\varepsilon)
$$

Because of the assumptions we can apply the implicit function theorem to (43) to obtain a $C^{1}$-function $\eta(\varepsilon)$ defined in a neighborhood of $\varepsilon=0$ satisfying (43) and such that (38) holds. Setting

$$
y_{j}(\varepsilon):=Y_{j}(\eta(\varepsilon), \varepsilon), \quad j=1,2
$$

we see that $y_{1}(\varepsilon), y_{2}(\varepsilon)$ are bounded $C^{1}$-solutions of (35) with $\eta=\eta(\varepsilon)$ such that $y_{1}(0)=0$, $y_{2}(0)=0$. Then we can write $\left.y_{1}(\varepsilon)=\varepsilon \tilde{y}_{1}(\varepsilon), y_{2}(\varepsilon)=\varepsilon \tilde{y}_{2}(\varepsilon)\right)$ for continuous $\tilde{y}_{1}(\varepsilon), \tilde{y}_{2}(\varepsilon) \in Y$ where

$$
\mathcal{F}\left(\varepsilon \tilde{y}_{j}(\varepsilon)\right)+\varepsilon H_{j}\left(\varepsilon \widetilde{y}_{1}(\varepsilon), \varepsilon \tilde{y}_{2}(\varepsilon), \eta(\varepsilon), \varepsilon\right)=0, \quad j=1,2 .
$$

Clearly (39) follows differentiating the above equality at $\varepsilon=0$. The proof is complete.

Remark 4.2 Note that, because of $\mathcal{M}(\bar{\eta})=0$, (39) is equivalent to

$$
P \mathcal{F}^{\prime}(0) \tilde{y}_{j}(0)+H_{j}(0,0,0, \bar{\eta}, 0)=0, \quad j=1,2,
$$

which has the unique solution

$$
\tilde{y}_{j}(0)=-\left[P \mathcal{F}^{\prime}(0)\right]^{-1} H_{j}(0,0,0, \bar{\eta}, 0), \quad j=1,2 .
$$

Now we apply Theorem 4.1 to (28) with $\mathcal{F}(y), H_{1}\left(y_{1}, y_{2}, \eta, \varepsilon\right), H_{2}\left(y_{1}, y_{2}, \eta, \varepsilon\right)$ as in (33), (34) and

$$
Y=C_{b}^{1}\left(\mathbb{R}, \mathbb{R}^{2}\right), \quad X=C_{b}^{0}\left(\mathbb{R}, \mathbb{R}^{2}\right), \quad \eta=(\alpha, \kappa) \in \mathbb{R} \times \mathbb{R}^{m},
$$

where $C_{b}^{k}\left(\mathbb{R}, \mathbb{R}^{2}\right)$ is the Banach space of $C^{k}$-functions bounded together with their derivatives with the usual sup-norm.

We already observed that $\mathcal{F}(0)=0$ and $\mathcal{N F}^{\prime}(0)=0$. Moreover,

$$
\mathcal{R F}^{\prime}(0)=\left\{x=\left(x_{1}, x_{2}\right) \in X \mid \int_{-\infty}^{\infty} \psi_{i}^{*}(s) x_{i}(s) d s=0, i=1,2\right\}
$$

where $\psi_{i}(s)$ have been defined in the previous Section 3. So $d=2$ and $m=3$. We recall, from [19], that $\psi_{j}(s)=\varphi(s) v_{j}(\theta(s))$ where $v_{j}(t)$ are solutions of the adjoint equation of (16):

$$
\left.\omega\left(x_{h}(t)\right) v^{\prime}=\frac{\omega^{\prime}\left(x_{h}(t)\right)^{*}}{\omega\left(x_{h}(t)\right)} F\left(x_{h}(t)\right)^{*} v-F^{\prime}\left(x_{h}(t)\right)^{*} v, \quad t \in\right] T_{-}, T_{+}[,
$$


and $v_{j}(0)=\mathcal{R} P_{-}^{*} \cap \mathcal{N} P_{+}^{*}$. Hence (37) reads

$$
\mathcal{M}(\alpha, \kappa)=\left(\begin{array}{l}
\int_{-\infty}^{\infty} \frac{\psi_{1}^{*}(s)}{\varphi(s)} G_{1}(\gamma(s), \gamma(s), \alpha+\theta(s), 0, \kappa) d s \\
\int_{-\infty}^{\infty} \frac{\psi(s)}{\varphi(s)} G_{1}(\gamma(s), \gamma(s), \alpha+\theta(s), 0, \kappa) d s \\
\int_{-\infty}^{\infty} \frac{\psi_{1}^{*}(s)}{\varphi(s)} G_{2}(\gamma(s), \gamma(s), \alpha+\theta(s), 0, \kappa) d s \\
\int_{-\infty}^{\infty} \frac{\psi_{2}^{*}(s)}{\varphi(s)} G_{2}(\gamma(s), \gamma(s), \alpha+\theta(s), 0, \kappa) d s
\end{array}\right)
$$

or passing to time $t=\theta(s)$ :

$$
\mathcal{M}(\alpha, \kappa)=\left(\begin{array}{l}
\int_{T_{-}}^{T_{+}} v_{1}^{*}(t) \frac{G_{1}\left(x_{h}(t), x_{h}(t), t+\alpha, 0, \kappa\right)}{\omega\left(x_{h}(t)\right)} d t \\
\int_{T_{-}}^{T_{+}} v_{2}^{*}(t) \frac{G_{1}\left(x_{h}(t), x_{h}(t), t+\alpha, 0, \kappa\right)}{\omega\left(x_{h}(t)\right)} d t \\
\int_{T_{+}}^{T_{+}} v_{1}^{*}(t) \frac{G_{2}\left(x_{h}(t), x_{h}(t), t+\alpha, 0, \kappa\right)}{\omega\left(h_{h}(t)\right)} d t \\
\int_{T_{-}}^{T_{+}} v_{2}^{*}(t) \frac{G_{2}\left(x_{h}(t), x_{h}(t), t+\alpha, 0, \kappa\right)}{\omega\left(x_{h}(t)\right)} d t
\end{array}\right) .
$$

A direct application of Theorem 4.1 gives the following.

Theorem 4.3 Let $m=3$ and $\mathcal{M}(\alpha, \kappa)$ be given as in (46) where $v_{1}(t), v_{2}(t)$ are two independent bounded solutions (on $\mathbb{R}$ ) of the adjoint equation (45). Suppose that $\bar{\alpha}$ and $\bar{\kappa}$ exist so that

$$
\mathcal{M}(\bar{\alpha}, \bar{\kappa})=0 \quad \text { and } \quad \frac{\partial \mathcal{M}}{\partial(\alpha, \kappa)}(\bar{\alpha}, \bar{\kappa}) \in G L(4, \mathbb{R})
$$

Then there exist $\bar{\varepsilon}>0, \rho>0$, unique $C^{1}$-functions $\alpha(\varepsilon)$ and $\kappa(\varepsilon)$ with $\alpha(0)=\bar{\alpha}$ and $\kappa(0)=\bar{\kappa}$, defined for $|\varepsilon|<\bar{\varepsilon}$, and a unique solution $\left(z_{1}(s, \varepsilon), z_{2}(s, \varepsilon)\right)$ of (24) with $\alpha=\alpha(\varepsilon), \kappa=\kappa(\varepsilon)$, $0<|\varepsilon|<\bar{\varepsilon}$, such that

$$
\sup _{s \in \mathbb{R}}\left|z_{j}(s, \varepsilon)-\gamma(s)\right| \varphi(s)^{-1}<\rho, \quad j=1,2 .
$$

Moreover,

$$
\sup _{s \in \mathbb{R}}\left|z_{j}(s, \varepsilon)-\gamma(s)\right| \varphi(s)^{-1}=O(\varepsilon), \quad j=1,2 .
$$

Remark 4.4 (i) Equation (48) implies

$$
z_{j}(s, \varepsilon)=\gamma(s)+\varepsilon \tilde{y}_{j}(s, \varepsilon) \varphi(s), \quad j=1,2,
$$

for $C^{0}$-functions $\tilde{y}_{j}(s, \varepsilon)$ with $\sup _{|\varepsilon| \leq \bar{\varepsilon}} \sup _{s \in \mathbb{R}}\left|\widetilde{y}_{j}(s, \varepsilon)\right|<\infty$. Then we have

$$
z_{j}(s, \varepsilon)=\gamma(s)+\varepsilon \tilde{y}_{j}(s, \varepsilon) \varphi(s)=\gamma(s)+\varepsilon \tilde{y}_{j}(s, 0) \varphi(s)+\varepsilon w_{j}(s, \varepsilon) \varphi(s)
$$

with $w_{j}(s, \varepsilon)=\tilde{y}_{j}(s, \varepsilon)-\tilde{y}_{j}(s, 0)$, so $\lim _{\varepsilon \rightarrow 0} \sup _{s \in \mathbb{R}}\left|w_{j}(s, \varepsilon)\right|=0$. Hence

$$
\lim _{\varepsilon \rightarrow 0} \sup _{s \in \mathbb{R}}\left|z_{j}(s, \varepsilon)-\gamma(s)-\varepsilon \widetilde{y}_{j}(s, 0) \varphi(s)\right| \varphi(s)^{-1} \varepsilon^{-1}=0, \quad j=1,2,
$$


which gives a first order approximation of $z_{j}(s, \varepsilon)$. Next, $\tilde{y}_{j}(s, 0)$ can be computed using $(44)$ adapted to this case. Hence $\widetilde{y}_{j}(s, 0)$ are bounded solutions of

$$
y_{j}^{\prime}=\left[F^{\prime}(\gamma(s))-\frac{F(\gamma(s)) \omega^{\prime}(\gamma(s))}{\omega(\gamma(s))}-\frac{\varphi^{\prime}(s)}{\varphi(s)}\right] y_{j}+\frac{1}{\varphi(s)} G_{j}(\gamma(s), \gamma(s), \theta(s)+\bar{\alpha}, \bar{\kappa}) .
$$

Since (36) has exponential dichotomies on both $\mathbb{R}_{+}$(with projection $P_{+}=0$ ) and $\mathbb{R}_{-}$(with projection $P_{-}=\mathbb{I}$ ) it follows that

$$
\tilde{y}_{j}(s, 0)= \begin{cases}-\int_{s}^{\infty} X(s) X^{-1}(z) \frac{1}{\varphi(z)} G_{j}(\gamma(z), \gamma(z), \theta(z)+\bar{\alpha}, \bar{\kappa}) d z & \text { for } s \geq 0 \\ \int_{-\infty}^{s} X(s) X^{-1}(z) \frac{1}{\varphi(z)} G_{j}(\gamma(z), \gamma(z), \theta(z)+\bar{\alpha}, \bar{\kappa}) d z & \text { for } s \leq 0\end{cases}
$$

$X(s)$ is the fundamental solution of (36). Note formulas (50) are well defined at $s=0$, i.e., $\tilde{y}_{j}\left(0^{-}, 0\right)=\tilde{y}_{j}\left(0^{+}, 0\right)$, due to the first assumption of (47). Next, passing to time $t=\theta(s)$ and taking $z_{j}(t):=\varphi\left(\theta^{-1}(t)\right) \widetilde{y}_{j}\left(\theta^{-1}(t), 0\right)$, we get

$$
\begin{aligned}
z_{j}(t) & =\int_{-\infty}^{\theta^{-1}(t)} \varphi\left(\theta^{-1}(t)\right) X\left(\theta^{-1}(t)\right) X^{-1}(z) \varphi(z)^{-1} G_{j}(\gamma(z), \gamma(z), \theta(z)+\bar{\alpha}, \bar{\kappa}) d z \\
& =\int_{T_{-}}^{t} \varphi\left(\theta^{-1}(t)\right) X\left(\theta^{-1}(t)\right) X^{-1}\left(\theta^{-1}(u)\right) \varphi\left(\theta^{-1}(u)\right)^{-1} \frac{G_{j}\left(x_{h}(u), x_{h}(u), u+\bar{\alpha}, \bar{\kappa}\right)}{\omega\left(x_{h}(u)\right)} d u
\end{aligned}
$$

for $T_{-}<t \leq 0$ and

$$
z_{j}(t)=-\int_{t}^{T_{+}} \varphi\left(\theta^{-1}(t)\right) X\left(\theta^{-1}(t)\right) X^{-1}\left(\theta^{-1}(u)\right) \varphi\left(\theta^{-1}(u)\right)^{-1} \frac{G_{j}\left(x_{h}(u), x_{h}(u), u+\bar{\alpha}, \bar{\kappa}\right)}{\omega\left(x_{h}(u)\right)} d u
$$

for $0 \leq t<T_{+}$. Note that $z_{j}(t)$ solves

$$
\omega\left(x_{h}(t)\right) z^{\prime}=F^{\prime}\left(x_{h}(t)\right) z-F\left(x_{h}(t)\right) \frac{\omega^{\prime}\left(x_{h}(t)\right) z}{\omega\left(x_{h}(t)\right)}+G_{j}\left(x_{h}(t), x_{h}(t), t+\bar{\alpha}, \bar{\kappa}\right)
$$

with $\sup _{t \in] T_{-}, T_{+}}\left|z_{j}(t)\right| \varphi\left(\theta^{-1}(t)\right)^{-1}<\infty$, and $\varphi\left(\theta^{-1}(t)\right) X\left(\theta^{-1}(t)\right)$ is a fundamental solution of (16).

(ii) Using (49), the functions $x_{j}(t, \varepsilon):=z_{j}\left(\theta^{-1}(t-\alpha(\varepsilon))\right.$, $\left.\varepsilon\right)$ are bounded solutions of (7) in the interval $] T_{-}+\alpha(\varepsilon), T_{+}+\alpha(\varepsilon)[$ such that

$$
\lim _{\varepsilon \rightarrow 0} \sup _{t \in] T_{-}, T_{+}[}\left|x_{j}(t+\alpha(\varepsilon), \varepsilon)-x_{h}(t)-\varepsilon z_{j}(t)\right| \varphi\left(\theta^{-1}(t)\right)^{-1} \varepsilon^{-1}=0 .
$$

Summarizing, we obtain the following corollary of Theorem 4.3.

Corollary 4.5 Let $m=3$ and $\mathcal{M}(\alpha, \kappa)$ be given as in (46) where $v_{1}(t), v_{2}(t)$ are two independent bounded solutions (on $\mathbb{R}$ ) of the adjoint equation (45). Suppose that $\bar{\alpha}$ and $\bar{\kappa}$ exist so that

$$
\mathcal{M}(\bar{\alpha}, \bar{\kappa})=0 \quad \text { and } \quad \frac{\partial \mathcal{M}}{\partial(\alpha, \kappa)}(\bar{\alpha}, \bar{\kappa}) \in G L(4, \mathbb{R}) .
$$

Then there exist $\bar{\varepsilon}>0, \rho>0$, unique $C^{1}$-functions $\alpha(\varepsilon)$ and $\kappa(\varepsilon)$ with $\alpha(0)=\bar{\alpha}$ and $\kappa(0)=\bar{\kappa}$, defined for $|\varepsilon|<\bar{\varepsilon}$, and a unique solution $\left(x_{1}(t, \varepsilon), x_{2}(t, \varepsilon)\right)$ of (7) with $\alpha=\alpha(\varepsilon), \kappa=\kappa(\varepsilon)$, 
$0<|\varepsilon|<\bar{\varepsilon}$, such that

$$
\sup _{T_{-}<t<T_{+}}\left|x_{j}(t+\alpha(\varepsilon), \varepsilon)-x_{h}(t)\right| \varphi\left(\theta^{-1}(t)\right)^{-1}<\rho, \quad j=1,2 .
$$

Moreover,

$$
\sup _{T_{-}<t<T_{+}}\left|x_{j}(t+\alpha(\varepsilon), \varepsilon)-x_{h}(t)\right| \varphi\left(\theta^{-1}(t)\right)^{-1}=O(\varepsilon), \quad j=1,2 .
$$

\section{Applications to RLC circuits}

In this section we study the coupled equations

$$
\begin{aligned}
& \left(u_{1}+u_{1}^{2}\right)^{\prime \prime}+\varepsilon \gamma_{1}\left(u_{1}+u_{1}^{2}\right)^{\prime}+u_{1}-\varepsilon \lambda\left(u_{2}+u_{2}^{2}\right)^{\prime \prime}+\varepsilon \sin t=0, \\
& \left(u_{2}+u_{2}^{2}\right)^{\prime \prime}+\varepsilon \gamma_{2}\left(u_{2}+u_{2}^{2}\right)^{\prime}+u_{2}-\varepsilon \lambda\left(u_{1}+u_{1}^{2}\right)^{\prime \prime}+\varepsilon \chi \sin (t+\varpi)=0,
\end{aligned}
$$

which is motivated by $[2,3]$. Note that (51) is obtained by coupling two equations modeling nonlinear RLC circuits as in (1). Here $\gamma_{1}, \gamma_{2}, \lambda, \chi$ and $\varpi$ are positive parameters. Setting $w_{j}=\left(u_{j}+u_{j}^{2}\right)^{\prime}, j=1,2$, (51) reads

$$
\left\{\begin{array}{l}
\left(2 u_{1}+1\right) u_{1}^{\prime}=w_{1} \\
w_{1}^{\prime}+\varepsilon \gamma_{1} w_{1}+u_{1}-\varepsilon \lambda w_{2}^{\prime}+\varepsilon \sin t=0 \\
\left(2 u_{2}+1\right) u_{2}^{\prime}=w_{2} \\
w_{2}^{\prime}+\varepsilon \gamma_{2} w_{2}+u_{2}-\varepsilon \lambda w_{1}^{\prime}+\varepsilon \chi \sin (t+\varpi)=0
\end{array}\right.
$$

By solving the second and fourth equations of (52) for $w_{1}^{\prime}$ and $w_{2}^{\prime}$, we get:

$$
\left\{\begin{array}{l}
\left(2 u_{1}+1\right) u_{1}^{\prime}=w_{1} \\
w_{1}^{\prime}=-u_{1}+\varepsilon \frac{\sin t+\lambda u_{2}+\gamma_{1} w_{1}+\varepsilon \lambda\left(\chi \sin (t+\infty)+\lambda u_{1}+\gamma_{2} w_{2}\right)}{\varepsilon^{2} \lambda^{2}-1} \\
\left(2 u_{2}+1\right) u_{2}^{\prime}=w_{2} \\
w_{2}^{\prime}=-u_{2}+\varepsilon \frac{\chi \sin (t+\varpi)+\lambda u_{1}+\gamma_{2} w_{2}+\varepsilon \lambda\left(\sin t+\lambda u_{2}+\gamma_{1} w_{1}\right)}{\varepsilon^{2} \lambda^{2}-1}
\end{array}\right.
$$

provided $|\varepsilon \lambda| \neq 1$. Since $\omega(u, w)=\omega(u)=2 u+1$, to write the system (52) in the form (7) with parameter $\kappa=\left(\gamma_{1}, \gamma_{2}, \lambda\right)$ and $(\chi, \varpi)$ fixed, we have to multiply the second and fourth equation by $2 u_{1,2}+1$, respectively, and we obtain the system

$$
\left\{\begin{array}{l}
\left(2 u_{1}+1\right) u_{1}^{\prime}=w_{1} \\
\left(2 u_{1}+1\right) w_{1}^{\prime}=-\left(2 u_{1}+1\right) u_{1}+\varepsilon\left(2 u_{1}+1\right) \frac{\sin t+\lambda u_{2}+\gamma_{1} w_{1}+\varepsilon \lambda\left(x \sin (t+\sigma)+\lambda u_{1}+\gamma_{2} w_{2}\right)}{\varepsilon^{2} \lambda^{2}-1} \\
\left(2 u_{2}+1\right) u_{2}^{\prime}=w_{2} \\
\left(2 u_{2}+1\right) w_{2}^{\prime}=-\left(2 u_{2}+1\right) u_{2}+\varepsilon\left(2 u_{2}+1\right) \frac{\chi \sin (t+\varpi)+\lambda u_{1}+\gamma_{2} w_{2}+\varepsilon \lambda\left(\sin t+\lambda u_{2}+\gamma_{1} w_{1}\right)}{\varepsilon^{2} \lambda^{2}-1}
\end{array}\right.
$$

with (uncoupled) unperturbed equation for $\varepsilon=0$ (see (8)):

$$
\left\{\begin{array}{l}
\left(2 u_{1}+1\right) u_{1}^{\prime}=w_{1} \\
\left(2 u_{1}+1\right) w_{1}^{\prime}=-\left(2 u_{1}+1\right) u_{1} \\
\left(2 u_{2}+1\right) u_{2}^{\prime}=w_{2} \\
\left(2 u_{2}+1\right) w_{2}^{\prime}=-\left(2 u_{2}+1\right) u_{2}
\end{array}\right.
$$


Neglecting left multiplicators $2 u+1\left(u=u_{1}, u_{2}\right)$ in (55), we obtain the following system (see condition (C2)):

$$
\left\{\begin{array}{l}
u_{1}^{\prime}=w_{1}, \\
w_{1}^{\prime}=-\left(2 u_{1}+1\right) u_{1}, \\
u_{2}^{\prime}=w_{2}, \\
w_{2}^{\prime}=-\left(2 u_{2}+1\right) u_{2} .
\end{array}\right.
$$

Clearly, condition $(\mathrm{C} 1)$ is satisfied with $x_{0}=\left(-\frac{1}{2}, 0\right)$ and

$$
F(x)=(w,-(2 u+1) u)^{*}, \quad \omega(x)=2 u+1, \quad x=(u, w)^{*} .
$$

The equation $u^{\prime \prime}+(2 u+1) u=0$ has the prime integral $u^{\prime 2}+\frac{4}{3} u^{3}+u^{2}$. A solution $u_{0}(s)$ satisfying $\lim _{s \rightarrow \infty} u_{0}(s)=-\frac{1}{2}$ has to satisfy $u^{\prime 2}+\frac{4}{3} u^{3}+u^{2}-\frac{1}{12}=0 \Leftrightarrow 3 u^{\prime 2}+(4 u-1)\left(u+\frac{1}{2}\right)^{2}=$ 0 with the solution

$$
u_{0}(s)=\frac{1}{4}\left(1-3 \tanh ^{2} \frac{s}{2}\right)
$$

bounded on $\mathbb{R}$. Hence

$$
\gamma(s)=\left(\frac{1}{4}\left(1-3 \tanh ^{2} \frac{s}{2}\right),-6 \operatorname{csch}^{3} s \sinh ^{4} \frac{s}{2}\right)^{*} .
$$

Note $\omega(\gamma(s))=2 u_{0}(s)+1=\frac{3}{2} \operatorname{sech}^{2} \frac{s}{2}>0$. From

$$
F^{\prime}\left(x_{0}\right)=\left(\begin{array}{ll}
0 & 1 \\
1 & 0
\end{array}\right)
$$

we get $\mu_{ \pm}= \pm 1$ and $\gamma_{ \pm}=(1, \pm 1)^{*}$. Since $\nabla \omega\left(x_{0}\right)=(2,0)^{*}$, we derive $\left\langle\nabla \omega\left(x_{0}\right), \gamma_{ \pm}\right\rangle=2>0$, and condition (C3) holds as well. Next, we have

$$
\begin{aligned}
& G_{1}\left(x_{1}, x_{2}, t, \varepsilon, \kappa\right)=\frac{2 u_{1}+1}{\varepsilon^{2} \lambda^{2}-1}\left(\begin{array}{c}
0 \\
\sin t+\lambda u_{2}+\gamma_{1} w_{1}+\varepsilon \lambda\left(\chi \sin (t+\varpi)+\lambda u_{1}+\gamma_{2} w_{2}\right)
\end{array}\right) \\
& G_{2}\left(x_{1}, x_{2}, t, \varepsilon, \kappa\right)=\frac{2 u_{2}+1}{\varepsilon^{2} \lambda^{2}-1}\left(\begin{array}{c}
0 \\
\chi \sin (t+\varpi)+\lambda u_{1}+\gamma_{2} w_{2}+\varepsilon \lambda\left(\sin t+\lambda u_{2}+\gamma_{1} w_{1}\right)
\end{array}\right) ;
\end{aligned}
$$

hence $G_{i}\left(x_{0}, x_{0}, t, \varepsilon, \kappa\right)=0$ and assumption $(\mathrm{C} 2)$ is also verified. Here $x_{i}=\left(u_{i}, w_{i}\right)^{*}, i=1,2$. Furthermore by (15)

$$
\theta(s)=\int_{0}^{s} \omega(\gamma(\tau)) d \tau=\int_{0}^{s} \frac{3}{2} \operatorname{sech}^{2} \frac{\tau}{2} d \tau=3 \tanh \frac{s}{2},
$$

so $T_{ \pm}= \pm 3$ and

$$
x_{h}(t)=\gamma\left(\theta^{-1}(t)\right)=\frac{1}{4}\left(1-\frac{t^{2}}{3}, t\left(\frac{t^{2}}{9}-1\right)\right)^{*} .
$$


Thus

$$
\begin{aligned}
& \omega\left(x_{h}(t)\right)=\frac{1}{6}\left(9-t^{2}\right), \quad \nabla \omega\left(x_{h}(t)\right)=(2,0)^{*} \\
& \frac{F\left(x_{h}(t)\right)}{\omega\left(x_{h}(t)\right)}=\frac{1}{12}\left(-2 t, t^{2}-3\right)^{*}, \quad F^{\prime}\left(x_{h}(t)\right)=\left(\begin{array}{cc}
0 & 1 \\
\frac{t^{2}}{3}-2 & 0
\end{array}\right) .
\end{aligned}
$$

So now (16) has the form

$$
\begin{aligned}
& z_{1}^{\prime}=\frac{2 t}{9-t^{2}} z_{1}+\frac{6}{9-t^{2}} z_{2}, \\
& z_{2}^{\prime}=-z_{1}
\end{aligned}
$$

which has the solution $x_{h}^{\prime}(t)=\left(-\frac{t}{6}, \frac{1}{12}\left(t^{2}-3\right)\right)$. In other words, we deal with

$$
z_{2}^{\prime \prime}=\frac{2 t}{9-t^{2}} z_{2}^{\prime}-\frac{6}{9-t^{2}} z_{2}
$$

possessing the solution $\frac{1}{12}\left(t^{2}-3\right)$. Following [27, p.327], the second solution of (58) is given by

$$
\frac{1}{9}\left(\left(t^{2}-3\right) \operatorname{arctanh} \frac{t}{3}-3 t\right)
$$

Consequently a fundamental matrix solution of (57) has the form

$$
Z(t)=\left(\begin{array}{cc}
-\frac{t}{6} & \frac{2}{9}\left(\frac{3\left(t^{2}-6\right)}{t^{2}-9}-t \operatorname{arctanh} \frac{t}{3}\right) \\
\frac{1}{12}\left(t^{2}-3\right) & \frac{1}{9}\left(t^{2}-3\right) \operatorname{arctanh} \frac{t}{3}-\frac{t}{3}
\end{array}\right)
$$

Note $\operatorname{det} Z(t)=\frac{1}{9-t^{2}}$. The adjoint system of $(57)$ is (see (45))

$$
\begin{aligned}
& \zeta_{1}^{\prime}=\frac{2 t}{t^{2}-9} \zeta_{1}+\zeta_{2}, \\
& \zeta_{2}^{\prime}=\frac{6}{t^{2}-9} \zeta_{1}
\end{aligned}
$$

with the fundamental matrix solution

$$
Z^{-1 *}(t)=\left(\begin{array}{cc}
\frac{1}{9}\left(9-t^{2}\right)\left(\left(t^{2}-3\right) \operatorname{arctanh} \frac{t}{3}-3 t\right) & \frac{1}{12}\left(t^{2}-9\right)\left(t^{2}-3\right) \\
\frac{2}{3}\left(t^{2}-6\right)-\frac{2}{9} t\left(t^{2}-9\right) \operatorname{arctanh} \frac{t}{3} & \frac{1}{6} t\left(t^{2}-9\right)
\end{array}\right)
$$

Note $\lim _{t \rightarrow \pm 3} Z^{-1 *}(t)=\left(\begin{array}{ll}0 & 0 \\ 2 & 0\end{array}\right)$. Thus we take

$$
v_{1}(t)=\left(\begin{array}{c}
\frac{1}{9}\left(t^{2}-9\right)\left(3 t-\left(t^{2}-3\right) \operatorname{arctanh} \frac{t}{3}\right) \\
\frac{2}{3}\left(t^{2}-6\right)-\frac{2}{9} t\left(t^{2}-9\right) \operatorname{arctanh} \frac{t}{3}
\end{array}\right)
$$

and

$$
v_{2}(t)=\left(\begin{array}{c}
\frac{1}{12}\left(t^{2}-9\right)\left(t^{2}-3\right) \\
\frac{1}{6} t\left(t^{2}-9\right)
\end{array}\right)
$$


We now compute $\mathcal{M}(\alpha, \kappa)$. We have (see the appendix)

$$
\begin{aligned}
& \int_{T_{-}}^{T_{+}} v_{1}^{*}(t) \frac{G_{1}\left(x_{h}(t), x_{h}(t), t+\alpha, 0, \kappa\right)}{\omega\left(x_{h}(t)\right)} d t \\
& =\frac{1}{3}(9 \lambda+4 \sin \alpha(\Gamma(2 \cos 3-3 \sin 3)+\cos 3(-9-2 \operatorname{Ci} 6+\log 36-3 \operatorname{Si} 6) \\
& \quad+\sin 3(3+3 \operatorname{Ci} 6-3 \log 6-2 \operatorname{Si} 6)))=a_{11} \lambda+a_{12} \sin \alpha,
\end{aligned}
$$

where $\operatorname{Si}(t)=\int_{0}^{t} \frac{\sin s}{s} d s$ is the sine integral function, $\operatorname{Ci}(t)=-\int_{t}^{\infty} \frac{\cos s}{s} d s$ is the cosine integral function $[28, \mathrm{p} .886]$ and $\Gamma$ is the Euler constant. Similarly

$$
\begin{aligned}
& \int_{T_{-}}^{T_{+}} v_{2}^{*}(t) \frac{G_{1}\left(x_{h}(t), x_{h}(t), t+\alpha, 0, \kappa\right)}{\omega\left(x_{h}(t)\right)} d t \\
& \quad=-\frac{54 \gamma_{1}}{35}-2 \cos \alpha(3 \cos 3+2 \sin 3)=a_{21} \gamma_{1}+a_{22} \cos \alpha
\end{aligned}
$$

and

$$
\begin{aligned}
& \int_{T_{-}}^{T_{+}} v_{1}^{*}(t) \frac{G_{2}\left(x_{h}(t), x_{h}(t), t+\alpha, 0, \kappa\right)}{\omega\left(x_{h}(t)\right)} d t=a_{11} \lambda+a_{12} \chi \sin (\alpha+\varpi), \\
& \int_{T_{-}}^{T_{+}} v_{2}^{*}(t) \frac{G_{2}\left(x_{h}(t), x_{h}(t), t+\alpha, 0, \kappa\right)}{\omega\left(x_{h}(t)\right)} d t=a_{21} \gamma_{2}+a_{22} \chi \cos (\alpha+\varpi) .
\end{aligned}
$$

Note

$$
a_{11}=3, \quad a_{12} \doteq 9.7406, \quad a_{21}=-\frac{54}{35}, \quad a_{22} \doteq 5.37547 .
$$

Consequently, the Melnikov function is now

$$
\mathcal{M}(\alpha, \kappa)=\left(\begin{array}{c}
a_{11} \lambda+a_{12} \sin \alpha \\
a_{21} \gamma_{1}+a_{22} \cos \alpha \\
a_{11} \lambda+a_{12} \chi \sin (\alpha+\varpi) \\
a_{21} \gamma_{2}+a_{22} \chi \cos (\alpha+\varpi)
\end{array}\right)
$$

The equation $\mathcal{M}(\alpha, \kappa)=0$ is equivalent to

$$
\begin{aligned}
& \lambda=-\frac{a_{12}}{a_{11}} \sin \alpha=-\frac{a_{12}}{a_{11}} \chi \sin (\alpha+\varpi), \\
& \gamma_{1}=-\frac{a_{22}}{a_{21}} \cos \alpha, \quad \gamma_{2}=-\frac{a_{22}}{a_{21}} \chi \cos (\alpha+\varpi) .
\end{aligned}
$$

So having $\chi>0$ and $\varpi>0$ such that the equation

$$
\sin \alpha-\chi \sin (\alpha+\varpi)=0
$$

has a simple zero $\alpha_{0}$ with $\sin \alpha_{0}<0, \cos \alpha_{0}>0$ and $\cos \left(\alpha_{0}+\varpi\right)>0$, formulas (64) give a simple zero $\left(\alpha_{0}, \gamma_{1,0}, \gamma_{2,0}, \lambda_{0}\right)$ of (63) with positive $\gamma_{1,0}, \gamma_{2,0}, \lambda_{0}$, and Corollary 4.5 can be 
applied to (51). If $\chi \cos \varpi \neq 1$, then (65) is equivalent to

$$
\tan \alpha=\frac{\chi \sin \varpi}{1-\chi \cos \varpi} .
$$

Hence assuming $\varpi \in] \pi, 2 \pi$ [ and $\chi \cos \varpi<1$, the right hand side of (66) is negative, and then

$$
\left.\alpha_{0}=\arctan \frac{\chi \sin \varpi}{1-\chi \cos \varpi} \in\right]-\frac{\pi}{2}, 0[
$$

satisfies $\sin \alpha_{0}<0$ and $\cos \alpha_{0}>0$. Since $\alpha_{0}$ satisfies (65) and $\sin \alpha_{0}<0$, condition $\cos \left(\alpha_{0}+\right.$ $\varpi)>0$ is equivalent to $\tan \left(\alpha_{0}+\varpi\right)<0$. Then using (66), we derive

$$
0>\tan \left(\alpha_{0}+\varpi\right)=\frac{\sin \varpi}{\cos \varpi-\chi}, \quad \varpi \neq \frac{3 \pi}{2} .
$$

When $\cos \varpi<0$, then (68) is not satisfied, since $\sin \varpi<0$. So we take $\varpi \in] \frac{3 \pi}{2}, 2 \pi$ [ and (68) gives also $\chi<\cos \varpi$. Clearly $\chi<\cos \varpi$ implies $1>\chi \cos \varpi$.

Summarizing we see that for any fixed $\chi$ and $\varpi$ satisfying

$$
0<\chi<\cos \varpi, \quad \varpi \in] \frac{3 \pi}{2}, 2 \pi[
$$

the Melnikov function (63) has a simple zero $\left(\alpha_{0}, \gamma_{1,0}, \gamma_{2,0}, \lambda_{0}\right)$ given by (64) and (67), and $\gamma_{1,0}>0, \gamma_{2,0}>0, \lambda_{0}>0$. Hence in the region given by (69) we apply Corollary 4.5 to (51) with parameters $\gamma_{1}, \gamma_{2}, \lambda$ near $\gamma_{1,0}, \gamma_{2,0}, \lambda_{0}$ determined by (64) and (67), i.e.,

$$
\begin{aligned}
& \lambda_{0}=-\frac{a_{12} \chi \sin \varpi}{a_{11} \sqrt{1+\chi^{2}-2 \chi \cos \varpi}}, \\
& \gamma_{1,0}=-\frac{a_{22}(1-\chi \cos \varpi)}{a_{21} \sqrt{1+\chi^{2}-2 \chi \cos \varpi}}, \\
& \gamma_{2,0}=-\chi \frac{a_{22}(\cos \varpi-\chi)}{a_{21} \sqrt{1+\chi^{2}-2 \chi \cos \varpi}}
\end{aligned}
$$

for any fixed $\varpi$ and $\chi$ satisfying (69). Summarizing, we get the following.

Theorem 5.1 For any fixed $\varpi, \chi$ satisfying (69) and then $\alpha_{0}, \gamma_{1,0}, \gamma_{2,0}, \lambda_{0}$ given by (67) and (70), there is an $\varepsilon_{0}>0$ and smooth functions $\left.\alpha, \gamma_{1}, \gamma_{2}, \lambda:\right]-\varepsilon_{0}, \varepsilon\left[\rightarrow \mathbb{R}\right.$ with $\alpha(0)=\alpha_{0}$, $\gamma_{1}(0)=\gamma_{1,0}, \gamma_{2}(0)=\gamma_{2,0}, \lambda(0)=\lambda_{0}$, such that for any $\left.\varepsilon \in\right]-\varepsilon_{0}, \varepsilon_{0}\left[\backslash\{0\}\right.$, system (51) with $\gamma_{1}=$ $\gamma_{1}(\varepsilon), \gamma_{2}=\gamma_{2}(\varepsilon), \lambda=\lambda(\varepsilon)$, possesses a unique solution $\left(u_{1}(\varepsilon, t), u_{2}(\varepsilon, t)\right)$ on $]-3+\alpha(\varepsilon), 3+$ $\alpha(\varepsilon)[$ such that

$$
\begin{aligned}
& \lim _{\varepsilon \rightarrow 0} \sup _{t \in]-3,3[}\left|u_{j}(\varepsilon, t+\alpha(\varepsilon))-\frac{1}{4}\left(1-\frac{t^{2}}{3}\right)\right|\left(9-t^{2}\right)^{-1}=0, \\
& \lim _{\varepsilon \rightarrow 0} \sup _{t \in]-3,3[}\left|u_{j}^{\prime}(\varepsilon, t+\alpha(\varepsilon))+\frac{t}{6}\right|=0 .
\end{aligned}
$$


Proof We apply Corollary 4.5 to (53). Since, in this case, $\varphi\left(\theta^{-1}(t)\right)=\frac{1}{2} \frac{9-t^{2}}{9+t^{2}}$, according to Corollary $4.5(53)$ has a solution $\left(u_{j}(t), w_{j}(t)\right), j=1,2$, such that

$$
\begin{aligned}
& \lim _{\varepsilon \rightarrow 0} \sup _{t \in]-3,3[}\left|u_{j}(\varepsilon, t+\alpha(\varepsilon))-\frac{1}{4}\left(1-\frac{t^{2}}{3}\right)\right|\left(9-t^{2}\right)^{-1}=0, \\
& \lim _{\varepsilon \rightarrow 0} \sup _{t \in]-3,3}\left|w_{j}(\varepsilon, t+\alpha(\varepsilon))-\frac{t}{4}\left(\frac{t^{2}}{9}-1\right)\right|\left(9-t^{2}\right)^{-1}=0 .
\end{aligned}
$$

Now, $w_{j}(\varepsilon, t)=\left(1+2 u_{j}(\varepsilon, t)\right) u_{j}^{\prime}(\varepsilon, t)$ and $\frac{t}{4}\left(\frac{t^{2}}{9}-1\right)=\left(1+2 u_{h}(t)\right) u_{h}^{\prime}(t)$, where $u_{h}(t)=\frac{1}{4}\left(1-\frac{t^{2}}{3}\right)$. So, taking $t^{\prime}:=t+\alpha(\varepsilon)$,

$$
\begin{aligned}
& w_{j}\left(\varepsilon, t^{\prime}\right)-\frac{t}{4}\left(\frac{t^{2}}{9}-1\right) \\
&=\left(1+2 u_{j}\left(\varepsilon, t^{\prime}\right)\right) u_{j}^{\prime}\left(\varepsilon, t^{\prime}\right)-\left(1+2 u_{h}(t)\right) u_{h}^{\prime}(t) \\
&=\left(1+2 u_{j}\left(\varepsilon, t^{\prime}\right)\right)\left(u_{j}^{\prime}\left(\varepsilon, t^{\prime}\right)-u_{h}^{\prime}(t)\right)+2\left(u_{j}\left(\varepsilon, t^{\prime}\right)-u_{h}(t)\right) u_{h}^{\prime}(t) \\
&=\left(1+2 u_{h}(t)\right)\left(u_{j}^{\prime}\left(\varepsilon, t^{\prime}\right)-u_{h}^{\prime}(t)\right)+2\left(u_{j}\left(\varepsilon, t^{\prime}\right)-u_{h}(t)\right)\left(u_{j}^{\prime}\left(\varepsilon, t^{\prime}\right)-u_{h}^{\prime}(t)\right) \\
&+2\left(u_{j}\left(\varepsilon, t^{\prime}\right)-u_{h}(t)\right) u_{h}^{\prime}(t) \\
&= {\left[\frac{9-t^{2}}{6}+2\left(u_{j}\left(\varepsilon, t^{\prime}\right)-u_{h}(t)\right)\right]\left(u_{j}^{\prime}\left(\varepsilon, t^{\prime}\right)-u_{h}^{\prime}(t)\right) } \\
&-\frac{t}{3}\left(u_{j}\left(\varepsilon, t^{\prime}\right)-u_{h}(t)\right)
\end{aligned}
$$

and then

$$
\begin{aligned}
& {\left[w_{j}\left(\varepsilon, t^{\prime}\right)-\frac{t}{4}\left(\frac{t^{2}}{9}-1\right)\right]\left(9-t^{2}\right)^{-1}} \\
& \quad=\left[\frac{1}{6}+2 \frac{u_{j}\left(\varepsilon, t^{\prime}\right)-u_{h}(t)}{9-t^{2}}\right]\left(u_{j}^{\prime}\left(\varepsilon, t^{\prime}\right)-u_{h}^{\prime}(t)\right)-\frac{t}{3} \frac{u_{j}\left(\varepsilon, t^{\prime}\right)-u_{h}(t)}{9-t^{2}},
\end{aligned}
$$

or, equivalently,

$$
\begin{aligned}
& {\left[\frac{1}{6}+2 \frac{u_{j}\left(\varepsilon, t^{\prime}\right)-u_{h}(t)}{9-t^{2}}\right]\left(u_{j}^{\prime}\left(\varepsilon, t^{\prime}\right)-u_{h}^{\prime}(t)\right)} \\
& \quad=\left[w_{j}\left(\varepsilon, t^{\prime}\right)-\frac{t}{4}\left(\frac{t^{2}}{9}-1\right)\right]\left(9-t^{2}\right)^{-1}+\frac{t}{3} \frac{u_{j}\left(\varepsilon, t^{\prime}\right)-u_{h}(t)}{9-t^{2}} .
\end{aligned}
$$

Since $\sup _{-3<t<3}\left|u_{j}\left(\varepsilon, t^{\prime}\right)-u_{h}(t)\right|\left(9-t^{2}\right)^{-1}=O(\varepsilon)$ we see that, for $\varepsilon$ sufficiently small,

$$
\frac{1}{7}<\left|\frac{1}{6}+2 \frac{u_{j}\left(\varepsilon, t^{\prime}\right)-u_{h}(t)}{9-t^{2}}\right|<\frac{1}{5}
$$

and hence, using (74) and (72),

$$
\lim _{\varepsilon \rightarrow 0} \sup _{-3<t<3}\left|u_{j}^{\prime}\left(\varepsilon, t^{\prime}\right)-u_{h}^{\prime}(t)\right|=0
$$


Vice versa, if (75) and the first of (72) hold, then (73) gives

$$
\lim _{\varepsilon \rightarrow 0} \sup _{-3<t<3}\left|w_{j}(\varepsilon, t+\alpha(\varepsilon))-\frac{t}{4}\left(\frac{t^{2}}{9}-1\right)\right|\left(9-t^{2}\right)^{-1}=0 .
$$

Hence (71) and (72) are equivalent. The proof is complete.

Of course, solutions given by Theorem 5.1 vary smoothly with respect $(\varpi, \chi)$ satisfying (69).

Remark 5.2 Missed in the above analysis is the second possibility when $\varpi \in] 0, \pi[$. Then $\sin \varpi>0$ and (66) is negative if

$$
\kappa \cos \varpi>1,
$$

so we get $\varpi \in] 0, \frac{\pi}{2}$ [ and $\kappa>1$. Then inequality of (68) is satisfied since $\kappa>1>\cos \varpi$. So we conclude that the result of Theorem 5.1 is valid also for

$$
\begin{aligned}
& \kappa \cos \varpi>1, \quad \varpi \in] 0, \frac{\pi}{2}[, \\
& \lambda_{0}=\frac{a_{12} \chi \sin \varpi}{a_{11} \sqrt{1+\chi^{2}-2 \chi \cos \varpi}}, \\
& \gamma_{1,0}=\frac{a_{22}(1-\chi \cos \varpi)}{a_{21} \sqrt{1+\chi^{2}-2 \chi \cos \varpi}}, \\
& \gamma_{2,0}=\chi \frac{a_{22}(\cos \varpi-\chi)}{a_{21} \sqrt{1+\chi^{2}-2 \chi \cos \varpi}} .
\end{aligned}
$$

\section{Appendix}

Let $v_{12}(t)=\frac{2}{3}\left(t^{2}-6\right)-\frac{2}{9} t\left(t^{2}-9\right) \operatorname{arctanh} \frac{t}{3}$ be the second component of $v_{1}(t)$. Note that $v_{12}(t)$ is an even function and then

$$
\begin{aligned}
& \int_{T_{-}}^{T_{+}} v_{1}^{*}(t) \frac{G_{1}\left(x_{h}(t), x_{h}(t), t+\alpha, 0, \kappa\right)}{\omega\left(x_{h}(t)\right)} d t \\
& \quad=-\int_{-3}^{3} v_{12}(t)\left[\gamma_{1} \frac{t}{4}\left(\frac{t^{2}}{9}-1\right)+\frac{\lambda}{4}\left(1-\frac{t^{2}}{3}\right)+\sin t \cos \alpha+\sin \alpha \cos t\right] d t \\
& \quad=-\int_{-3}^{3} v_{12}(t)\left[\frac{\lambda}{4}\left(1-\frac{t^{2}}{3}\right)+\sin \alpha \cos t\right] d t \\
& \quad=\frac{\lambda}{12} \int_{-3}^{3} v_{12}(t)\left(t^{2}-3\right) d t-\int_{-3}^{3} v_{12}(t) \cos t d t \sin \alpha .
\end{aligned}
$$

Similarly,

$$
\begin{aligned}
& \int_{T_{-}}^{T_{+}} v_{1}^{*}(t) \frac{G_{2}\left(x_{h}(t), x_{h}(t), t+\alpha, 0, \kappa\right)}{\omega\left(x_{h}(t)\right)} d t \\
& \quad=\frac{\lambda}{12} \int_{-3}^{3} v_{12}(t)\left(t^{2}-3\right) d t-\chi \int_{-3}^{3} v_{12}(t) \cos t d t \sin (\alpha+\varpi) .
\end{aligned}
$$


Now, using $\lim _{t \rightarrow \pm 1^{\mp}}\left(t^{2}-1\right) \operatorname{arctanh}(t)=0$ and integration by parts of the second integral,

$$
\begin{aligned}
& \int_{-3}^{3} v_{12}(t)\left(t^{2}-3\right) d t \\
& \quad=\int_{-3}^{3}\left[\frac{2}{3}\left(t^{2}-6\right)-\frac{2}{9} t\left(t^{2}-9\right) \operatorname{arctanh} \frac{t}{3}\right]\left(t^{2}-3\right) d t \\
& \quad=\frac{144}{5}-\frac{2}{9} \int_{-3}^{3} t\left(t^{2}-9\right)\left(t^{2}-3\right) \operatorname{arctanh} \frac{t}{3} d t \\
& \quad=\frac{144}{5}-\frac{2}{9}\left[\frac{t^{2}\left(t^{2}-9\right)^{2}}{6} \operatorname{arctanh} \frac{t}{3}-\int \frac{t^{2}\left(t^{2}-9\right)^{2}}{6} \frac{3}{9-t^{2}} d t\right]_{-3}^{3} \\
& =\frac{144}{5}-\frac{1}{9} \int_{-3}^{3} t^{2}\left(t^{2}-9\right) d t=\frac{144}{5}+\frac{36}{5}=36
\end{aligned}
$$

Furthermore, since

$$
\int_{-3}^{3} v_{12}(t) \cos t d t=\int_{-3}^{3}\left[\frac{2}{3}\left(t^{2}-6\right)-\frac{2}{9} t\left(t^{2}-9\right) \operatorname{arctanh} \frac{t}{3}\right] \cos t d t
$$

we compute

$$
\int_{-3}^{3}\left(t^{2}-6\right) \cos t d t=2(\sin 3+6 \cos 3)
$$

and

$$
\begin{aligned}
I(t):= & \int t\left(t^{2}-9\right) \cos t \operatorname{arctanh} \frac{t}{3} d t \\
= & \left(3\left(-5+t^{2}\right) \cos t+t\left(-15+t^{2}\right) \sin t\right) \operatorname{arctanh} \frac{t}{3} \\
& +\int \frac{3\left(3\left(-5+t^{2}\right) \cos t+t\left(-15+t^{2}\right) \sin t\right)}{-9+t^{2}} d t .
\end{aligned}
$$

Next

$$
\begin{aligned}
\int & \frac{3\left(-5+t^{2}\right) \cos t+t\left(-15+t^{2}\right) \sin t}{-9+t^{2}} d t \\
& =\int(3 \cos t+t \sin t) d t-6 \int \frac{-2 \cos t+t \sin t}{-9+t^{2}} d t \\
& =-t \cos t+4 \sin t-6 \int \frac{-2 \cos t+t \sin t}{-9+t^{2}} d t
\end{aligned}
$$

Furthermore,

$$
\begin{aligned}
\int \frac{\cos t}{-9+t^{2}} d t & =\frac{1}{6} \int \frac{\cos t}{t-3} d t-\frac{1}{6} \int \frac{\cos t}{t+3} d t \\
& =\left.\frac{1}{6} \int \frac{\cos (3-z)}{z} d z\right|_{z=3-t}-\left.\frac{1}{6} \int \frac{\cos (v-3)}{v} d v\right|_{\nu=t+3} \\
& =\frac{1}{6}\left(\left.\int \frac{\cos 3 \cos z+\sin 3 \sin z}{z} d z\right|_{z=3-t}\right.
\end{aligned}
$$




$$
\begin{aligned}
& \left.-\left.\int \frac{\cos v \cos 3+\sin v \sin 3}{v} d v\right|_{v=t+3}\right) \\
& =\frac{1}{6}(\cos 3 \mathrm{Ci}(3-t)+\sin 3 \operatorname{Si}(3-t))-\frac{1}{6}(\cos 3 \mathrm{Ci}(3+t)+\sin 3 \mathrm{Si}(3+t)) \\
& =\frac{1}{6}(\cos 3(\mathrm{Ci}(3-t)-\mathrm{Ci}(3+t))+\sin 3(\operatorname{Si}(3-t)-\operatorname{Si}(3+t))),
\end{aligned}
$$

and

$$
\begin{aligned}
\int \frac{t \sin t}{-9+t^{2}} d t & =\frac{1}{2} \int \frac{\sin t}{t-3} d t+\frac{1}{2} \int \frac{\sin t}{t+3} d t \\
& =\frac{1}{2}(\sin 3 \operatorname{Ci}(3-t)-\cos 3 \operatorname{Si}(3-t))+\frac{1}{2}(\cos 3 \operatorname{Si}(3+t)-\sin 3 \operatorname{Ci}(3+t)) \\
& =\frac{1}{2}(\cos 3(\operatorname{Si}(3+t)-\operatorname{Si}(3-t))+\sin 3(\operatorname{Ci}(3-t)-\operatorname{Ci}(3+t)))
\end{aligned}
$$

Hence

$$
\begin{aligned}
I(t)= & \left(3\left(-5+t^{2}\right) \cos t+t\left(-15+t^{2}\right) \sin t\right) \operatorname{arctanh} \frac{t}{3}-3 t \cos t+12 \sin t \\
& +3(\mathrm{Ci}(3-t)(2 \cos 3-3 \sin 3)+\mathrm{Ci}(3+t)(-2 \cos 3+3 \sin 3) \\
& +(3 \cos 3+2 \sin 3)(\operatorname{Si}(3-t)-\operatorname{Si}(3+t)))
\end{aligned}
$$

Clearly $I(0)=0$. Next, using $\operatorname{arctanh} \frac{t}{3}=\frac{1}{2}(\ln 6-\ln (3-t))+o(1), \mathrm{Ci}(3-t)=\Gamma+\ln (3-t)+o(1)$ as $t \rightarrow 3_{-}$, we derive

$$
\begin{aligned}
I(t)= & \operatorname{Ci}(3+t)(-6 \cos 3+9 \sin 3)+\frac{1}{2}\left(3 \cos t\left(-2 t+2 o(1)\left(-5+t^{2}\right)+\left(-5+t^{2}\right) \ln 6\right)\right. \\
& +6(\Gamma+o(1))(2 \cos 3-3 \sin 3)+\left(24+t\left(-15+t^{2}\right)(2 o(1)+\ln 6)\right) \sin t \\
& +6(3 \cos 3+2 \sin 3)(\operatorname{Si}(3-t)-\operatorname{Si}(3+t))) \\
& +\frac{1}{2}\left(-3\left(-5+t^{2}\right) \cos t+6(2 \cos 3-3 \sin 3)-t\left(-15+t^{2}\right) \sin t\right) \ln (3-t) .
\end{aligned}
$$

Setting $f(t):=\frac{1}{2}\left(-3\left(-5+t^{2}\right) \cos t+6(2 \cos 3-3 \sin 3)-t\left(-15+t^{2}\right) \sin t\right)$ we see $f(3)=0$, so $f(t) \ln (3-t)=f^{\prime}\left(t^{\prime}\right)(t-3) \ln (3-t), t^{\prime} \in(t, 3)$ and using $\lim _{t \rightarrow 3_{-}}(t-3) \ln (3-t)=0$, we obtain

$$
\begin{aligned}
I(3-)= & \lim _{t \rightarrow 3-} I(t)=3 \cos 3(-3+2 \Gamma-2 \mathrm{Ci} 6+\ln 36-3 \operatorname{Si} 6) \\
& -3 \sin 3(-4+3 \Gamma-3 \mathrm{Ci} 6+\ln 216+2 \operatorname{Si} 6)
\end{aligned}
$$

Summarizing we arrive at

$$
\begin{aligned}
& \int_{-3}^{3} v_{12}(t) \cos t d t \\
& \quad=\frac{4}{3}(\sin 3+6 \cos 3)-\frac{4}{9} I(3-) \\
& \quad=\sin 3(-3+3 \Gamma-3 \mathrm{Ci} 6+\ln 216+2 \operatorname{Si} 6)+\cos 3(9-2 \Gamma+2 \mathrm{Ci} 6-\ln 36+3 \operatorname{Si} 6) .
\end{aligned}
$$


So inserting the above computations into (77) and (78), we get (60) and the first formula of (62). Next, since the second component $v_{22}(t)=\frac{1}{6} t\left(t^{2}-9\right)$ of $v_{2}(t)$ is odd, we get

$$
\begin{aligned}
& \int_{T_{-}}^{T_{+}} v_{2}^{*}(t) \frac{G_{1}\left(x_{h}(t), x_{h}(t), t+\alpha, 0, \kappa\right)}{\omega\left(x_{h}(t)\right)} d t \\
& =-\int_{-3}^{3} v_{22}(t)\left(\sin (t+\alpha)+\frac{\lambda}{4}\left(1-\frac{t^{2}}{3}\right)+\frac{\gamma_{1}}{4} t\left(\frac{t^{2}}{9}-1\right)\right) d t \\
& \quad=-\int_{-3}^{3} v_{22}(t) \sin t d t \cos \alpha+\frac{1}{36} \gamma_{1} \int_{-3}^{3} v_{22}(t) t\left(9-t^{2}\right) d t .
\end{aligned}
$$

But

$$
\int_{-3}^{3} v_{22}(t) t\left(9-t^{2}\right) d t=-\int_{-3}^{3} \frac{1}{6} t^{2}\left(t^{2}-9\right)^{2} d t=-\frac{1944}{35}
$$

and

$$
\int_{-3}^{3} v_{22}(t) \sin t d t=\int_{-3}^{3} \frac{1}{6} t\left(t^{2}-9\right) \sin t d t=4 \sin 3+6 \cos 3
$$

So we obtain (61). Similarly,

$$
\begin{aligned}
& \int_{T_{-}}^{T_{+}} v_{2}^{*}(t) \frac{G_{2}\left(x_{h}(t), x_{h}(t), t+\alpha, 0, \kappa\right)}{\omega\left(x_{h}(t)\right)} d t \\
& \quad=-\int_{-3}^{3} v_{22}(t)\left(\chi \sin (t+\alpha+\varpi)+\frac{\lambda}{4}\left(1-\frac{t^{2}}{3}\right)+\frac{\gamma_{2}}{4} t\left(\frac{t^{2}}{9}-1\right)\right) d t \\
& =-\int_{-3}^{3} v_{22}(t) \sin t d t \cos (\alpha+\varpi)+\frac{1}{36} \gamma_{2} \int_{-3}^{3} v_{22}(t) t\left(9-t^{2}\right) d t \\
& =-(4 \sin 3+6 \cos 3) \cos (\alpha+\varpi)-\frac{54}{35} \gamma_{2},
\end{aligned}
$$

which implies the second formula of (62).

\section{Competing interests}

The authors declare that they have no competing interests.

\section{Authors' contributions}

The work presented here was carried out in collaboration between the authors. The authors contributed to every part of this study equally and read and approved the final version of the manuscript.

\section{Author details}

'Department of Industrial Engineering and Mathematical Sciences, Marche Polytecnic University, Via Brecce Bianche 1, Ancona, 60131, Italy. ${ }^{2}$ Department of Mathematical Analysis and Numerical Mathematics, Comenius University, Mlynská dolina, Bratislava, 842 48, Slovakia. ${ }^{3}$ Mathematical Institute of Slovak Academy of Sciences, Štefánikova 49, Bratislava, 814 73, Slovakia.

\section{Acknowledgements}

BF is partially supported by PRIN-MURST 'Equazioni Differenziali Ordinarie e Applicazioni' (Italy). MF is partially supported by the Grants VEGA-MS 1/0071/14, VEGA-SAV 2/0029/13 and Marche Polytechnic University, Ancona (Italy).

Received: 18 December 2013 Accepted: 1 April 2014 Published: 07 May 2014

\section{References}

1. Battelli, F, Fečkan, M: Nonlinear RLC circuits and implicit ode's. Differ. Integral Equ. (2014) 
2. Lazarides, N, Eleftheriou, M, Tsironis, GP: Discrete breathers in nonlinear magnetic metamaterials. Phys. Rev. Lett. 97, $157406(2006)$

3. Veldes, GP, Cuevas, J, Kevrekidis, PG, Frantzeskakis, DJ: Quasidiscrete microwave solitons in a split-ring-resonator-based left-handed coplanar waveguide. Phys. Rev. E 83, 046608 (2011)

4. Riaza, R: Differential-Algebraic Systems, Analytical Aspects and Circuit Applications. World Scientific, Singapore (2008)

5. Kunkel, P, Mehrmann, V: Differential-Algebraic Equations, Analysis and Numerical Solution. Eur. Math. Soc., Zürich (2006)

6. Medved', M: Normal forms of implicit and observed implicit differential equations. Riv. Mat. Pura Appl. 10, 95-107 (1991)

7. Medved', M: Qualitative properties of generalized vector fields. Riv. Mat. Pura Appl. 15, 7-31 (1994)

8. Rabier, PJ: Implicit differential equations near a singular point. J. Math. Anal. Appl. 144, 425-449 (1989)

9. Rabier, PJ, Rheinboldt, WC: A general existence and uniqueness theorem for implicit differential algebraic equations. Differ. Integral Equ. 4, 563-582 (1991)

10. Rabier, PJ, Rheinbold, WC: A geometric treatment of implicit differential-algebraic equations. J. Differ. Equ. 109, 110-146 (1994)

11. Rabier, PJ, Rheinbold, WC: On impasse points of quasilinear differential algebraic equations. J. Math. Anal. Appl. 181, 429-454 (1994)

12. Rabier, PJ, Rheinbold, WC: On the computation of impasse points of quasilinear differential algebraic equations. Math. Comput. 62, 133-154 (1994)

13. Andres, J, Górniewicz, L: Topological Principles for Boundary Value Problems. Kluwer, Dordrecht (2003)

14. Górniewicz, L: Topological Fixed Point Theory of Multivalued Mappings. Springer, Berlin (2009)

15. Fečkan, M: Existence results for implicit differential equations. Math. Slovaca 48, 35-42 (1998)

16. Frigon, M, Kaczynski, T: Boundary value problems for systems of implicit differential equations. J. Math. Anal. Appl. $179,317-326(1993)$

17. Heikkilä, S, Kumpulainen, M, Seikkala, S: Uniqueness and comparison results for implicit differential equations. Dyn. Syst. Appl. 7, 237-244 (1998)

18. Li, D: Peano's theorem for implicit differential equations. J. Math. Anal. Appl. 258, 591-616 (2001)

19. Battelli, F, Fečkan, M: Melnikov theory for nonlinear implicit ode's. J. Differ. Equ. 256, 1157-1190 (2014)

20. Awrejcewicz, J, Holicke, MM: Melnikov's method and stick-slip chaotic oscillations in very weekly forced mechanical systems. Int. J. Bifurc. Chaos 9, 505-518 (1999)

21. Awrejcewicz, J, Pyryev, Y: Chaos prediction in the Duffing type system with friction using Melnikov's functions. Nonlinear Anal., Real World Appl. 7, 12-24 (2006)

22. Coddington, EA, Levinson, N: Theory of Ordinary Differential Equations. Tata McGraw-Hill, New Delhi (1972). Reprint (1987)

23. Battelli, F, Lazzari, C: Exponential dichotomies, heteroclinic orbits, and Melnikov functions. J. Differ. Equ. 86, 342-366 (1990)

24. Coppel, WA: Dichotomies in Stability Theory. Springer, Berlin (1978)

25. Palmer, KJ: Exponential dichotomies and transversal homoclinic points. J. Differ. Equ. 55, 225-256 (1984)

26. Calamai, A, Franca, M: Melnikov methods and homoclinic orbits in discontinuous systems. J. Dyn. Differ. Equ. 25 733-764 (2013)

27. Hartman, P: Ordinary Differential Equations. Wiley, New York (1964)

28. Gradshteyn, IS, Ryzhik, IM: Table of Integrals, Series, and Products. Academic Press, Boston (2007)

10.1186/1687-2770-2014-101

Cite this article as: Battelli and Fečkan: Melnikov theory for weakly coupled nonlinear RLC circuits. Boundary Value Problems 2014, 2014:101

\section{Submit your manuscript to a SpringerOpen ${ }^{\circ}$ journal and benefit from:}

- Convenient online submission

- Rigorous peer review

Immediate publication on acceptance

- Open access: articles freely available online

- High visibility within the field

- Retaining the copyright to your article 